\title{
Spectra of Structures and Relations
}

\author{
Valentina S. Harizanov Russell G. Miller ${ }^{\dagger}$
}

June 14, 2006

\begin{abstract}
We consider embeddings of structures which preserve spectra: if $g$ : $\mathcal{M} \rightarrow \mathcal{S}$ with $\mathcal{S}$ computable, then $\mathcal{M}$ should have the same Turing degree spectrum (as a structure) that $g(\mathcal{M})$ has (as a relation on $\mathcal{S}$ ). We show that the computable dense linear order $\mathcal{L}$ is universal for all countable linear orders under this notion of embedding, and we establish a similar result for the computable random graph $\mathcal{G}$. Such structures are said to be spectrally universal. We use our results to answer a question of Goncharov, and also to characterize the possible spectra of structures as precisely the spectra of unary relations on $\mathcal{G}$. Finally, we consider the extent to which all spectra of unary relations on the structure $\mathcal{L}$ may be realized by such embeddings, offering partial results and building the first known example of a structure whose spectrum contains precisely those degrees $\mathbf{c}$ with $\mathbf{c}^{\prime} \geq_{\mathbf{T}} \mathbf{0}^{\prime \prime}$.
\end{abstract}

\section{Introduction}

In model theory, a model $\mathcal{S}$ of a theory $T$ is said to be universal for $T$ if every model $\mathcal{M}$ of $T$ of cardinality $\leq|\mathcal{S}|$ embeds into $\mathcal{S}$. Common examples are the countable dense linear order without end points (for the theory of linear orders) and the countable atomless Boolean algebra (for the theory of Boolean algebras).

From the standpoint of computability theory, we wish to define a more restrictive notion of universality, requiring not just the existence of embeddings $g$ from each $\mathcal{M}$ into $\mathcal{S}$, but also that these embeddings preserve computabilitytheoretic properties of the structures. In particular, we are interested in the spectrum of the structure $\mathcal{M}$, and the degree spectrum of its image $g(\mathcal{M})$ as a subset of $\mathcal{S}$.

\footnotetext{
$\dagger$ The first author was partially supported by the NSF under grant DMS-0502499. The first author also wishes to thank William Frawley for establishing the Columbian Research Fellowship of the George Washington University, which generously supported her research. The second author was partially supported by a VIGRE postdoc under NSF grant number 9983660 to Cornell University, and by grants number 60095-34-35 and 80209-04-12 from The City University of New York PSC-CUNY Research Award Program. The authors also wish to acknowledge useful conversations with Barbara Csima, Denis Hirschfeldt, Antonio Montalbán, Richard Shore, and Reed Solomon.
} 
The Turing degree of a countable structure $\mathcal{M}$ with domain $\omega$ is the Turing degree of its atomic diagram. If the language is finite, this is the join of the degrees of the different functions $f^{\mathcal{M}}$ and relations $R^{\mathcal{M}}$, where $f$ and $R$ range over all function and relation symbols in the language of $\mathcal{M}$. (We will assume in this paper that the language is finite, unless otherwise stated.) By definition, the spectrum of (the isomorphism type of) $\mathcal{M}$ is the set of all Turing degrees of isomorphic copies of $\mathcal{M}$ :

$$
\operatorname{Spec}(\mathcal{M})=\{\operatorname{deg}(\mathcal{N}): \mathcal{N} \cong \mathcal{M}\} .
$$

Intuitively, this measures the intrinsic difficulty of computing a copy of $\mathcal{M}$ : each degree $\mathbf{d}$ in $\operatorname{Spec}(\mathcal{M})$ is smart enough to build a structure isomorphic to $\mathcal{M}$. Conversely, for $\mathbf{d}$ to lie in $\operatorname{Spec}(\mathcal{M}), \mathcal{M}$ must be complicated enough to allow some way of coding $\mathbf{d}$ into a copy of $\mathcal{M}$. As seen in Theorem 1.4 below, the requirement of being "smart enough" is usually the difficult one when we ask whether $\mathbf{d}$ lies in $\operatorname{Spec}(\mathcal{M})$; coding is possible in all but certain trivial cases.

On the other hand, the degree spectrum of a relation $R$ on a computable structure $\mathcal{A}$ is defined as:

$$
\operatorname{DgSp}_{\mathcal{A}}(R)=\left\{\operatorname{deg}(S):\left(\exists \mathcal{B} \leq_{T} \emptyset\right)(\mathcal{B}, S) \cong(\mathcal{A}, R)\right\}
$$

(By convention, for relations one speaks of the "degree spectrum" rather than the "spectrum." There seems to be no good reason for this distinction in terminology, but we will not attempt here to unify the terms.) The symbol $R$ generally is not in the language of the structure $\mathcal{A}$; indeed, if it were, then $\operatorname{DgSp}_{\mathcal{A}}(R)$ would contain only $\mathbf{0}$.

Again, the intuition we wish to capture by defining the degree spectrum of $R$ is the question of how complicated we can make the relation $R$. Of course, if the definition allowed $\mathcal{B}$ to be any isomorphic copy of $\mathcal{A}$, then we would have much more freedom to increase the complexity of the image $S$ of $R$ under the isomorphism from $\mathcal{A}$ to $\mathcal{B}$. Restricting the definition to computable structures $\mathcal{B}$ is our way of ruling out such tricks: for a degree $\mathbf{d}$ to lie in $\operatorname{DgSp}_{\mathcal{A}}(R)$, we must be able to make the image of $R$ have degree $\mathbf{d}$ while keeping the underlying structure computable.

Our goal in considering the notion of universality is to preserve and relate these two notions of the spectrum:

Definition 1.1 We say that a computable model $\mathcal{S}$ of a theory $T$ is spectrally universal if for every countable nontrivial model $\mathcal{M}$ of $T$, there exists an embedding $g: \mathcal{M} \rightarrow \mathcal{S}$ such that

$$
\operatorname{DgSp}_{\mathcal{S}}(g(\mathcal{M}))=\operatorname{Spec}(\mathcal{M})
$$

(Trivial models are defined on p. 4 below, where we will see the reasons for excluding them.) Thus, the embeddings we seek must preserve the spectrum of each $\mathcal{M}$, mapping it into $\mathcal{S}$ in such a way that its image has precisely the same complexity (as measured by our notions of spectra) as the original structure $\mathcal{M}$. 
In this paper we prove that the computable dense linear order $\mathcal{L}$ (without end points) is spectrally universal for the theory of linear orders, and that the computable random graph $\mathcal{G}$ is spectrally universal for the theory of (symmetric irreflexive) graphs. We will build specific computable copies $\mathcal{L}$ and $\mathcal{G}$ of these structures to help simplify our proofs, but by computable categoricity (as described below), every computable copy of $\mathcal{L}$ and $\mathcal{G}$ will be spectrally universal for the respective theory.

The two models $\mathcal{L}$ and $\mathcal{G}$ actually satisfy a stronger version of spectral universality, in that for each of them one can give a computable function $f$ with the following property: if $\mathcal{M}=\left(\omega, \Phi_{e}^{C}\right)$ is a model of the relevant theory with $\operatorname{deg}(\mathcal{M})=\operatorname{deg}(C)$, then the oracle function $\Phi_{f(e)}^{C}$ serves as the embedding $g$ described above. (Since each of the two relevant languages contains a single binary relation symbol, the oracle and one index $e$ are all that is required to describe $\mathcal{M}$.) This is a uniform version of spectral universality, in that aside from an $\mathcal{M}$-oracle and the indices for the functions and relations of $\mathcal{M}$, we need no special information about $\mathcal{M}$. Indeed, for every $\mathcal{A}=\left(\omega, \Phi_{i}^{D}\right)$ isomorphic to $\mathcal{M}$, with $D \equiv_{T} \mathcal{A}$, we will have $\left(\mathcal{S}, \Phi_{f(e)}^{C}(\mathcal{M})\right) \cong\left(\mathcal{S}, \Phi_{f(i)}^{D}(\mathcal{A})\right)$ and $\Phi_{f(i)}^{D}(\mathcal{A}) \equiv_{T} \mathcal{A}$. These two properties together are essentially all that is needed to prove spectral universality of $\mathcal{S}$. It remains an open question whether there are spectrally universal structures for which this uniform version fails.

The following lemma is immediate from Definition 1.1.

Lemma 1.2 If $\mathcal{S}$ is spectrally universal for a theory $T$, then for every model $\mathcal{M}$ of $T$, there is a unary relation $R$ on $\mathcal{S}$ such that $D g S p_{\mathcal{S}}(R)=\operatorname{Spec}(\mathcal{M})$.

Thus, a spectrally universal model $\mathcal{S}$ of $T$ can use results about the possible spectra of models of $T$ to help classify the possible degree spectra of relations on $\mathcal{S}$, or vice versa. Indeed, the genesis of this paper was a question asked by Goncharov:

Question 1.3 (Goncharov) Does there exist a relation $R$ on a computable linear order $\mathcal{A}$ such that

$$
D g S p_{\mathcal{A}}(R) \cap \Delta_{2}^{0}=\left\{\mathbf{d} \leq_{\mathbf{T}} \mathbf{0}^{\prime}: \mathbf{d} \neq \mathbf{0}\right\} ?
$$

In Corollary 2.3, we give a positive answer, using the construction in [17] of a linear order whose spectrum has the desired property.

We note that ordinarily the spectra of structures and the degree spectra of relations need not be related in such ways. Degree spectra can easily have upper bounds under Turing reducibility; for instance, if $R$ is $\Sigma_{n}^{0}$-definable in $\mathcal{A}$, then clearly every degree in $\operatorname{DgSp}_{\mathcal{A}}(R)$ will be $\Sigma_{n}^{0}$, and similarly for $\Pi_{n^{-}}^{0}$ definable relations. In fact, Downey had already proven the existence of a computable linear order $\mathcal{B}$ and a relation $R$ such that $\operatorname{DgSp}_{\mathcal{B}}(R)$ contained all non-computable c.e. degrees but not $\mathbf{0}$. He applied Lemma 1.2 from [6] to the linear order $\mathcal{A}$ built in [17], yielding a computable linear order $\mathcal{B}$, and proved that the degree spectrum of the adjacency relation on $\mathcal{B}$ contains precisely those c.e. degrees which lie in $\operatorname{Spec}(\mathcal{A})$, that is, all c.e. degrees except $\mathbf{0}$. However, since 
the adjacency relation is $\Pi_{1}^{0}$-definable, its degree spectrum clearly cannot contain any degrees that are not c.e. Many results are known about the relationship between definability of a relation and upper bounds on its degree spectrum; we refer the reader to [9] for details.

In contrast, if the spectrum of a structure has an upper bound under Turing reducibility, then that spectrum can only contain a single degree. For this result, we remind the reader of the following theorem of Knight from [16].

Theorem 1.4 (Knight) In any computable language, let $\mathcal{A}$ be a structure whose domain is an initial segment of $\omega$. Then exactly one of the following two statements holds:

- For any two Turing degrees $\mathbf{c} \leq_{\mathbf{T}} \mathbf{d}$, if $\mathbf{c} \in \operatorname{Spec}(\mathcal{A})$, then also $\mathbf{d} \in \operatorname{Spec}(\mathcal{A})$. (That is, the spectrum of $\mathcal{A}$ is upward-closed under $\leq_{T}$.)

- There exists a finite set $\left\{a_{1}, \ldots, a_{n}\right\}$ in the domain of $\mathcal{A}$ such that every permutation $f$ of $\omega$ with $f\left(a_{i}\right)=a_{i}$ for $i \leq n$ is an automorphism of $\mathcal{A}$.

In computable model thory, structures satisfying the second of these statements are called trivial; they include all finite structures, of course, and also some infinite structures, such as the complete graph on countably many vertices. The following corollary of Theorem 1.4 is quickly seen.

Corollary 1.5 In a finite language, let $\mathcal{A}$ be a structure with domain $\omega$. Then $\mathcal{A}$ is trivial if and only if its spectrum is $\{\mathbf{0}\}$.

The spectrum of a trivial structure always contains exactly one Turing degree, but if the language is infinite, that degree can be noncomputable. In this paper we use only finite languages, and so the exclusion of trivial structures in Definition 1.1 removes only one very simple possible spectrum from consideration. For finite structures, every embedding preserves the spectrum, of course, but for infinite trivial structures it can be difficult to preserve the spectrum, even when it is possible for all nontrivial structures. Since we regard trivial structures as anomalies anyway, we excluded them when defining spectral universality.

Proof of Corollary 1.5. The backwards implication is immediate from Theorem 1.4. For the forwards implication, let $\mathcal{A}$ be trivial, and choose a set $S=\left\{a_{1}, \ldots, a_{n}\right\}$ from its domain to satisfy the definition of triviality. We will show that $\mathcal{A}$ is computable.

If $R_{1}$ is a unary relation symbol in the language, fix any $x \notin S$. For each $y \notin S$, let $g_{y}$ be the permutation of $\omega$ which permutes $x$ and $y$ and fixes all other points. Then we have

$$
R_{1}^{\mathcal{A}}(y) \Longleftrightarrow R_{1}^{\mathcal{A}}\left(g_{y}(x)\right) \Longleftrightarrow R_{1}^{\mathcal{A}}(x)
$$

since $g_{y}$ must be an automorphism of $\mathcal{A}$. Thus $R_{1}^{\mathcal{A}}$ is computable. Similarly, for a binary $R_{2}$, we fix distinct $x_{1}, x_{2} \notin S$, and note that for every distinct pair 
$y_{1}, y_{2} \notin S$ and every $i \leq n$ we have

$$
\begin{aligned}
& R_{2}^{\mathcal{A}}\left(y_{1}, y_{2}\right) \Longleftrightarrow R_{2}^{\mathcal{A}}\left(x_{1}, x_{2}\right), \\
& R_{2}^{\mathcal{A}}\left(y_{1}, y_{1}\right) \Longleftrightarrow R_{2}^{\mathcal{A}}\left(x_{1}, x_{1}\right), \\
& R_{2}^{\mathcal{A}}\left(y_{1}, a_{i}\right) \Longleftrightarrow R_{2}^{\mathcal{A}}\left(x_{1}, a_{i}\right), \\
& R_{2}^{\mathcal{A}}\left(a_{i}, y_{2}\right) \Longleftrightarrow R_{2}^{\mathcal{A}}\left(a_{i}, x_{2}\right),
\end{aligned}
$$

by applying automorphisms which permute $x_{1}$ with $y_{1}$ and $x_{2}$ with $y_{2}$. (A moment's thought is required when $x_{1}=y_{2}$ or $x_{2}=y_{1}$, but the result still holds.) So $R_{2}^{\mathcal{A}}$ is computable, and it is clear how to extend this argument to any $k$-ary relation.

If $f$ is a unary function symbol, $x \notin S$ is fixed, and $y \notin S \cup\left\{x, f^{\mathcal{A}}(x)\right\}$ is arbitrary, let $g_{y}$ be as above. Then

$$
f^{\mathcal{A}}(y)=f^{\mathcal{A}}\left(g_{y}(x)\right)=g_{y}\left(f^{\mathcal{A}}(x)\right) .
$$

If $f^{\mathcal{A}}(x)=x$, then $f^{\mathcal{A}}$ is the identity on $\bar{S}$; otherwise it is constant there. Either way $f^{\mathcal{A}}$ is computable. The argument for $k$-ary functions is left to the reader; they are always finite unions of projections and constant functions on computable disjoint subsets of $\omega^{k}$.

At certain points we will use the concept of computable categoricity to simplify our arguments. A computable structure $\mathcal{A}$ is computably categorical if for every computable structure $\mathcal{B}$ isomorphic to $\mathcal{A}$, there exists a computable isomorphism from $\mathcal{B}$ onto $\mathcal{A}$. We have a similar (but strictly stronger) notion for structures that need not be computable: $\mathcal{A}$ is relatively computably categorical if for every $\mathcal{B}$ isomorphic to $\mathcal{A}$, there exists an isomorphism from $\mathcal{B}$ onto $\mathcal{A}$ which is computable in the join of $\operatorname{deg}(\mathcal{A})$ and $\operatorname{deg}(\mathcal{B})$. (In some of the literature, this notion is defined only for computable structures $\mathcal{A}$, but it makes sense for any countable structure.)

The subjects of the remaining sections are the countable dense linear order $\mathcal{L}$, the random graph $\mathcal{G}$, and (to a lesser extent) the countable atomless Boolean algebra $\mathcal{B}$, all of which are relatively computably categorical, hence computably categorical. Indeed, the classical model-theoretic arguments for $\omega$-categoricity of their theories are effective, and therefore build isomorphisms computable in the degrees of the structures. These concepts are useful here for the following reason.

Lemma 1.6 Let $\mathcal{A}$ be computably categorical, and $R$ a relation on $\mathcal{A}$. Then for every degree $\mathbf{d}$ in $D_{g S p_{\mathcal{A}}}(R)$, there exists a relation $S$ on $\mathcal{A}$ itself such that $(\mathcal{A}, R) \cong(\mathcal{A}, S)$ and $S \in \mathbf{d}$.

Thus we need not consider other computable copies of $\mathcal{A}$ when dealing with the degree spectrum of $R$.

Proof. Since $\mathbf{d}$ lies in $\operatorname{DgSp}_{\mathcal{A}}(R)$, we have a computable $\mathcal{B}$ isomorphic to $\mathcal{A}$, and a relation $T$ of degree $\mathbf{d}$ such that $(\mathcal{A}, R) \cong(\mathcal{B}, T)$. By computable categoricity, there is a computable isomorphism $f$ taking $\mathcal{B}$ onto $\mathcal{A}$. Let $S=f(T)$. 
A good reference for computable categoricity is [1]. For questions about notation, we refer the reader to [20], the standard source.

\section{Linear Orders}

Let $\prec$ be a computable linear order on $\omega$ such that $(\omega, \prec) \cong \eta$, the countable dense linear order without end points, and let $\theta:(\omega, \prec) \rightarrow(\mathbb{Q},<)$ be a computable isomorphism onto the rational numbers. (Formally, this $\theta$ consists of two computable functions $g(n)$ and $h(n)$ giving the numerator and denominator of $\theta(n)$, with $h(n)>0$ and $g(n) \in \mathbb{Z}$.) We write $\mathcal{L}=(\omega, \prec)$.

We will use standard notation $(a, b)$ and $[a, b]$ for open and closed intervals. Sometimes we will adjoin a subscript to remind the reader which structure the interval lies in, e.g., $(a, b)_{\mathcal{L}}$ for an open $\prec$-interval in $\mathcal{L}$, or $[\theta(a), \theta(b)]_{\mathbb{Q}}$ for a closed $<$-interval in $\mathbb{Q}$.

Theorem 2.1 This structure $\mathcal{L}$ is spectrally universal for the theory of linear orders.

Corollary 2.2 Let $\mathcal{A}$ be any countable linear order. Then there exists a unary relation $R$ on $\mathcal{L}$ such that

$$
\operatorname{DgSp} p_{\mathcal{L}}(R)=\operatorname{Spec}(\mathcal{A})
$$

In [17], Miller constructed a linear order $\mathcal{A}$ such that $\operatorname{Spec}(\mathcal{A}) \cap \Delta_{2}^{0}=$ $\Delta_{2}^{0}-\{\mathbf{0}\}$. Using Corollary 2.2, this yields a positive answer to Question 1.3 of Goncharov:

Corollary 2.3 There exists a computable linear order $\mathcal{L}$ with a relation $R$ such that

$$
D g S p_{\mathcal{L}}(R) \cap \Delta_{2}^{0}=\Delta_{2}^{0}-\{\mathbf{0}\} .
$$

Proof of Theorem 2.1. As part of the proof, we will need to work with a specific subset of the digits of our rationals. Define $d_{n}=3 n$ and

$$
c_{n}= \begin{cases}1, & \text { if }(\exists k) n=\frac{k(k+1)}{2} \\ 0, & \text { otherwise }\end{cases}
$$

for all $n$. Thus $\left\langle c_{n}\right\rangle$ is the sequence $1101001000100001 \ldots$. Also, we define

$$
\begin{aligned}
& J_{n}=\left\{q \in \mathbb{Q} \cap[0,1]:(\forall \text { representations } h \text { of } q) h\left(d_{n}\right)=c_{n}\right\} \\
& I_{n}=\cap_{m \leq n} J_{m} .
\end{aligned}
$$

(A binary sequence $h \in 2^{\omega}$ represents a real $r \in[0,1]$ if $\sum_{n} \frac{h(n)}{2^{n+1}}=r$.) Since the $(3 n)$-th digit is fixed, each $J_{n}$ comprises $2^{(3 n)}=8^{n}$ distinct open intervals in $\mathbb{Q}$, exactly $4^{n}$ of which lie in $I_{n}$. These $4^{n}$ intervals are the components of $I_{n}$. 
We will arrange that for every $n$, all but finitely many elements of the image $R$ of the embedding $g$ lie in $I_{n}$. Therefore, if a sequence of rationals in $R$ converges to a real $h$, then for each $n$, cofinitely many rationals in the sequence must belong to $I_{n}$ (and hence cofinitely many lie in some single component of $I_{n}$, by convergence). Hence $h\left(d_{n}\right)=c_{n}$ for all $n$, so that $h$ cannot be rational. (Since $c_{m}=1$ for some $m>n, h$ cannot be the left end point of its component in $I_{n}$; similarly for the right end point.)

Construction: The trivial linear orders are precisely the finite ones, so fix any countable infinite linear order $\mathcal{A}$, and let $\mathcal{B}$ be any copy of $\mathcal{A}$ with domain $\omega$. Set $\mathbf{c}=\operatorname{deg}(\mathcal{B})$, so $\mathbf{c} \in \operatorname{Spec}(\mathcal{A})$. Pick a set $C \in \mathbf{c}$ to serve as an oracle for $\mathcal{B}$. We will build an embedding $g: \mathcal{B} \rightarrow \mathcal{L}$ as required by Definition 1.1.

Let $\mathcal{B}_{s}$ be the restriction of $\mathcal{B}$ to the elements $\{0, \ldots, s-1\}$. We begin by fixing the elements $p$ and $p^{\prime}$ in $\omega$ such that $\theta(p)=0$ and $\theta\left(p^{\prime}\right)=1$ (using the $\theta$ from p. 6), and we define $l(p)=0$ and $l\left(p^{\prime}\right)=1$. The embedding $g$ will map $\mathcal{B}$ into the interval $\left(p, p^{\prime}\right)$ in $\mathcal{L}$. We now build $R \leq_{T} C$, the image of $g$ in $\mathcal{L}$, starting with $R_{0}=\emptyset$, so that $(R, \prec \uparrow(R \times R)) \cong \mathcal{B}$.

At stage $s+1, \mathcal{B}_{s}$ contains exactly $s$ elements, mapped by $g$ to corresponding elements $q_{i} \in R_{s}$, say with $p \prec q_{1} \prec \cdots \prec q_{s} \prec p^{\prime}$. Set $q_{0}=p$ and $q_{s+1}=p^{\prime}$ for convenience, and suppose that the element $s$ added to $\mathcal{B}$ at stage $s+1$ becomes the $(i+1)$-st element of $\mathcal{B}_{s+1}$, so that we wish to define $g(s)$ to be an element from $\left(q_{i}, q_{i+1}\right)_{\mathcal{L}}$. (Here we use the oracle $C$ to compute the order on $\mathcal{B}$.) We define the target set $I$ as follows.

If $l\left(q_{i}\right)>l\left(q_{i+1}\right)$, let $l=1+l\left(q_{i}\right)$. By induction, $\theta\left(q_{i}\right)$ will lie in some particular component $J$ within $I_{l-1}$. Moreover, of the four components of $I_{l}$ within this $J, \theta\left(q_{i}\right)$ will lie within one of the two central ones. Now the rightmost component of $I_{l}$ within $J$ contains in turn four components of $I_{l+1}$, and we define the target set $I$ to be the union of the two central of these four components. Thus $I \subset I_{l+1}$. Below we will enumerate into $R$ an $x$ such that $\theta(x) \in I$. This will ensure that $\theta(x)$ and $\theta\left(q_{i}\right)$ lie in distinct components of $I_{l}$, though in the same component of $I_{l-1}$, allowing us to prove Lemma 2.5 by induction.

If $l\left(q_{i}\right)<l\left(q_{i+1}\right)$, let $l=1+l\left(q_{i+1}\right)$. Analogously to the preceding paragraph, we let $J$ be the component of $I_{l-1}$ containing $\theta\left(q_{i+1}\right)$, and consider the leftmost component of $I_{l}$ within $J$. The target set $I$ is now defined as the union of the central two components of $I_{l+1}$ within this leftmost component of $I_{l}$ in $J$. Again $I \subset I_{l}$, therefore, and $\theta(x)$ and $\theta\left(q_{i+1}\right)$ will lie in distinct components of $I_{l}$ within the same component of $I_{l-1}$.

The following diagram illustrates the situation when $l\left(q_{i}\right)>l\left(q_{i+1}\right)$.

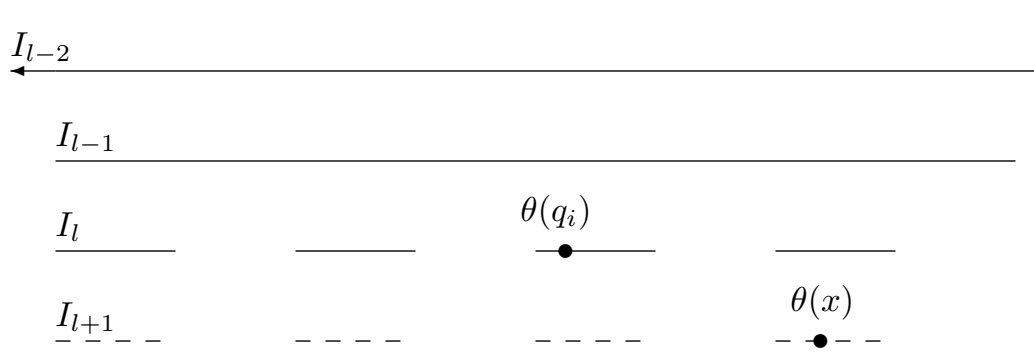


We search for the least $x \in \omega$ such that $\theta(x) \in I$ and $x>s$. Clearly such an $x$ must exist, and we define $g(s)=x$, enumerate $x \in R_{s+1}$ (so $R$ is still the image of $g)$, and set $l(x)=1+\max \left(l\left(q_{i}\right), l\left(q_{i+1}\right)\right)$. This completes the construction.

Notice that choosing $x$ in the target set $I$ guarantees that $q_{i} \prec x \prec q_{i+1}$. If $l\left(q_{i}\right)>l\left(q_{i+1}\right)$, for instance, then $q_{i} \prec x$ because $I$ is contained within a component of $I_{l}$ to the right of the component of $I_{l}$ in which $\theta\left(q_{i}\right)$ lies. Moreover, by induction, $\theta\left(q_{i}\right)$ and $\theta\left(q_{i+1}\right)$ cannot lie in the same component of $I_{l-1}$; instead $\theta\left(q_{i+1}\right)$ will lie to the right of $\theta\left(q_{i}\right)$ 's component, because $q_{i} \prec q_{i+1}$. Since $I$ is contained in the same component of $I_{l-1}$ as $\theta\left(q_{i}\right), I$ must be completely to the left of $\theta\left(q_{i+1}\right)$, so $x \prec q_{i+1}$. A similar argument applies when $l\left(q_{i+1}\right)>l\left(q_{i}\right)$, so the map $g$ which we have built is an order-isomorphism of $\mathcal{B}$ onto $(R, \prec \uparrow(R \times R))$.

The next two lemmas describe two useful properties of $R$.

Lemma 2.4 For every $n$, all but finitely many $r \in R$ satisfy $\theta(r) \in I_{n}$.

Proof. We have $l(p)=0$ and $l\left(p^{\prime}\right)=1$, with every $r \in R$ satisfying $p \prec r \prec p^{\prime}$. By induction, whenever an $x$ enters $R$ with $q_{i} \prec x \prec q_{i+1}$ (using the notation of the construction), we have $l(x)=1+\max \left(l\left(q_{i}\right), l\left(q_{i+1}\right)\right)$. Hence the first element $x$ to enter $R$ is the only one with $l(x)=2$, and if there are only $k$ elements $x$ with $l(x) \leq n$, then there can be at most $k+1$ many more $y \in R$ with $l(y)=n+1$, for once one such $y$ is placed in the interval between two such $x$, every subsequent element $z$ from that interval to enter $R$ will have $l(z)>l(y)$. Now it is clear from the construction that if $l(x) \geq n$, then $\theta(x) \in I_{n+1}$. The lemma follows.

Lemma 2.5 Let $\left\langle r_{i}\right\rangle_{i \geq 0}$ and $\left\langle t_{i}\right\rangle_{i \geq 0}$ be sequences of elements of $R$, strictly increasing and strictly decreasing (respectively). Then neither $\sup _{i} \theta\left(r_{i}\right)$ nor $\inf _{i} \theta\left(t_{i}\right)$ is a rational number.

Corollary 2.6 Under the order topology, $R$ is a discrete subset of $\mathcal{L}$. That is, no limit point of $R$ (in $\mathcal{L}$ ) lies in $R$.

Recall that a limit point of a set $S \subset \mathbb{R}$ is a point $u$ such that every open interval containing $u$ intersects $(S-\{u\})$.

Proof of Lemma 2.5. We give the details for the increasing sequence $r_{0} \prec r_{1} \prec$ $\cdots$. Since all $r_{i} \prec p^{\prime}$, the completeness of the reals yields a number $u=$ $\sup _{i} \theta\left(r_{i}\right) \in \mathbb{R}$. Since $r_{i} \prec r_{i+1}$, we know that $u \neq \theta\left(r_{i}\right)$ for all $i$. But by Lemma 2.4 , for each $n$, cofinitely many $\theta\left(r_{i}\right)$ must lie in $I_{n}$. Indeed, since $I_{n}$ consists of finitely many open components, one of these components $J$ must contain cofinitely many $\theta\left(r_{i}\right)$. Therefore $u$ must lie in the closure of $J$.

Keeping this $n$ fixed, we pick $m>n$ such that $c_{m}=0$. Now cofinitely many $\theta\left(r_{i}\right)$ must lie in $I_{m}$ as well, so cofinitely many lie in $I_{m} \cap J$. However, the rightmost component of $I_{m}$ within $J$ has its right end point within $J$, because $c_{m}=0$. Hence $u$ cannot be the right end point of $J$, and certainly $u$ cannot be the left end point of $J$, because all $\theta\left(r_{i}\right) \prec u$ and most $\theta\left(r_{i}\right)$ lie in $J$. So we have $u \in J \subset I_{n}$. 
Since this holds for every $n$, every binary expansion of $u$ has $c_{n}$ as its $d_{n}$-th digit for every $n$. But since $d_{n}=3 n$ and the sequence of $c_{n}$ 's is nonrepeating, $u$ cannot be rational.

With these two lemmas we can proceed to the heart of our proof.

Lemma 2.7 Let $\mathcal{B}$ and $\tilde{\mathcal{B}}$ be two copies of $\mathcal{A}$, of Turing degrees $\mathbf{c}$ and $\tilde{\mathbf{c}}$, respectively. Pick any sets $C \in \mathbf{c}$ and $\tilde{C} \in \tilde{\mathbf{c}}$, and run the preceding construction to produce embeddings $g$ and $\tilde{g}$ with images $R$ and $\tilde{R}$. Then $(\omega, \prec, R) \cong(\omega, \prec, \tilde{R})$.

Proof. Since $g$ is an embedding, the restriction of $\prec$ to $R$ gives a linear order isomorphic to $\mathcal{A}$, and similarly for $\tilde{R}$. So there exists a $\prec$-isomorphism $\rho$ : $R \rightarrow \tilde{R}$. We will extend this (non-computably, of course!) to the required isomorphism from $(\omega, \prec, R)$ to $(\omega, \prec, \tilde{R})$. Immediately we may define $\rho$ to be the identity on the closed intervals $(-\infty, p]$ and $\left[p^{\prime},+\infty\right)$.

Next, for each successivity of $\mathcal{A}$, the corresponding elements $q \prec r$ of $R$ bound an open interval $(q, r)$ under $\prec$ which is entirely contained in $\bar{R}$, and is itself a dense order without end points. We extend $\rho$ to map $(q, r)$ isomorphically onto the interval $(\rho(q), \rho(r))$, for which the same properties must hold.

If $q \in R$ has no immediate $R$-successor, then we let $r_{0}=p^{\prime}$ and let $r_{i+1}$ be the first element of the interval $\left(q, r_{i}\right)$ to appear in $R$. If $u=\inf _{i} \theta\left(r_{i}\right)$ in $\mathbb{R}$, then clearly $\theta(q) \leq u$, and by Lemma 2.5 we know that $u \notin \mathbb{Q}$, so $\theta(q)<u$. Thus $\left\{x \in \omega:(\forall i)\left[q \prec x \prec r_{i}\right]\right\}$ is a non-empty open interval, and so must be a dense order without end points. The same arguments apply to $\rho(q) \in \tilde{R}$, yielding another dense open interval of $\mathcal{L}$ with left end point $\rho(q)$ and irrational right end point, and we extend $\rho$ to map the open interval $\left(q, \theta^{-1}(u)\right)$ of $\bar{R}$ isomorphically onto the corresponding interval in $\bar{R}$.

For those $q \in R$ with no immediate $R$-predecessor, we apply the analogous process to extend $\rho$ to the open interval $\{x \prec q:(\forall r \in R)[r \prec q \Longrightarrow r \prec x]\}$, mapping it onto the corresponding interval of $\tilde{\tilde{R}}$. Again, Lemma 2.5 ensures that both these open intervals are non-empty, hence dense without end points.

We apply this same process with $q=p$. If $R$ has a left end point $r$, it must be $\succ p$, and we extend $\rho$ to map the interval $(p, r)$ isomorphically onto the interval $(p, \rho(r))$. If $R$ has no left end point, then by the same argument as above, $u=\inf \theta(R)$ is irrational, as is $\tilde{u}=\inf \theta(\tilde{R})$. So $\theta(p)<u$ and $\theta(p)<\tilde{u}$, and we extend $\rho$ to map $\{x \succ p: \theta(x)<u\}$ isomorphically onto $\{x \succ p: \theta(x)<\tilde{u}\}$. A similar argument extends $\rho$ to the interval with right end point $p^{\prime}$.

Now we claim that we have extended $\rho$ to all of $\omega$, and that $\rho$ is a $\prec-$ isomorphism onto $\omega$. (Also, our extensions so far clearly guarantee that $\rho(R)=$ $\tilde{R}$.) Pick any $x \in \bar{R}$. If $x$ has either an immediate $R$-predecessor or an immediate $R$-successor (or both), then $\rho(x)$ has been defined using that information. Suppose, therefore, that $x$ has neither. We use the same process as above to build sequences $p=r_{0} \prec r_{1} \prec \cdots$ and $p^{\prime}=t_{0} \succ t_{1} \succ \cdots$ such that $r_{i} \prec x \prec t_{i}$ for all $i$ and for every $r \in R$, either $r \prec r_{i}$ or $r \succ t_{i}$ for some $i$. (It is important that these sequences be chosen as above: $r_{i+1}$ is the first element of the interval $\left(r_{i}, x\right)$ to appear in $R$, and similarly for $t_{i+1}$.) We let $u=\sup _{i} \theta\left(r_{i}\right)$ and 
$v=\inf _{i} \theta\left(t_{i}\right)$. By Lemma 2.5, both $u$ and $v$ must be irrational, and we claim that in fact $u=v$. This will prove that there was no such $x$, since we would have to have $u \leq \theta(x) \leq v$.

We fix any positive integer $l$ and show that $v-u<8^{-l}$. Now $l\left(t_{i+1}\right)>l\left(t_{i}\right)$ for every $i$, by our choice of the sequence $\left\langle t_{i}\right\rangle$, so we fix some $j$ with $l\left(t_{j}\right) \geq l$ such that some $r_{i}$ enters $R$ at a stage $s$ with $t_{j} \in R_{s}$ and $t_{j+1} \notin R_{s}$. At stage $s$, therefore, our construction picked $r_{i}$ between $r_{i-1}$ and $t_{j}$. If $l\left(r_{i-1}\right)<l\left(t_{j}\right)$, then $r_{i}$ was chosen so that $\theta\left(r_{i}\right)$ and $\theta\left(t_{j}\right)$ lie in the same component of $I_{l\left(t_{j}\right)}$. These components each have length $8^{-l\left(t_{j}\right)-1}$, so $\theta\left(t_{j}\right)-\theta\left(r_{i}\right)<8^{-l}$. On the other hand, if $l\left(r_{i-1}\right)>l\left(t_{j}\right)$, then we wait for a stage $t>s$ at which $t_{j+1}$ enters $R$. Suppose $r_{k} \in R_{t}$ but $r_{k+1} \notin R_{t}$. Then $l\left(r_{k}\right)>l\left(r_{i-1}\right)>l\left(t_{j}\right)$, so the construction picks $t_{j+1}$ so that $\theta\left(t_{j+1}\right)$ lies in the same component of $I_{l\left(r_{k}\right)}$ as $\theta\left(r_{k}\right)$. Thus $\theta\left(t_{j+1}\right)-\theta\left(r_{k}\right)<8^{-l\left(r_{k}\right)-1}<8^{-l}$. As promised, therefore, we must have $v-u<8^{-l}$ for every $l$, and so $v=u$.

The same holds in the construction of $\tilde{R}$, of course, so the $\rho$ we have built is total and onto, and is indeed an isomorphism from $(\omega, \prec, R)$ onto $(\omega, \prec, \tilde{R})$.

Now we claim that our construction of $R$ ensured $R \leq_{T} C$. To determine whether $n \in R$, use the $C$-oracle to run this construction through stage $n$, since only elements $>n$ were allowed to enter $R$ after stage $n$. Thus $R(n)=$ $R_{n}(n)$, and so $R \leq_{T} C$. Theorem 2.10 below will allow us to conclude that $\mathbf{c} \in \operatorname{DgSp}_{\mathcal{L}}(R)$. (Corollary 2.6 shows that the infinite set $R$ is not a finite union of intervals in $\mathcal{L}$, so $R$ satisfies (2) of Theorem 2.10.) In fact, it is not difficult to modify the foregoing construction to code the oracle set $C \in \mathbf{c}$ into $R$, so that we could actually build $R \equiv_{T} C$. Alternatively, one can continue with the construction from the proof of Theorem 2.10 to get $R \equiv_{T} C$ uniformly in the $C$-oracle. This justifies the claims of uniformity made on page 3 .

Similarly, for any set $\tilde{C}$ in any other degree $\tilde{\mathbf{c}} \in \operatorname{Spec}(\mathcal{A})$, we have built $\tilde{R} \equiv_{T} \tilde{C}$ with $(\omega, \prec, R) \cong(\omega, \prec, \tilde{R})$, by Lemma 2.7 . Thus $\operatorname{Spec}(\mathcal{A}) \subseteq \operatorname{DgSp}_{\mathcal{L}}(R)$.

To see that $\operatorname{Spec}(\mathcal{A}) \supseteq \operatorname{DgSp}_{\mathcal{L}}(R)$, we suppose that $S$ is a unary relation such that $(\omega, \prec, R) \cong(\omega, \prec, S)$. (By Lemma 1.6, we need not consider other computable copies of $\mathcal{L}$.) Then the structure $(S, \prec \uparrow(S \times S))$ is a copy of $\mathcal{A}$. Say $S=\left\{x_{0}<x_{1}<\cdots\right\}$, and let $f(n)=x_{n}$. Then $f \leq_{T} S$, and the structure $(\omega, L)$, with $L(m, n)$ just if $f(m) \prec f(n)$, is a copy of $\mathcal{A}$ of degree $\leq_{T} S$. Since $\mathcal{A}$ is nontrivial, Theorem 1.4 shows that $\operatorname{Spec}(\mathcal{A})$ is closed upward under $\leq_{T}$, so $\operatorname{deg}(S) \in \operatorname{Spec}(\mathcal{A})$. (If $\mathcal{A}$ were trivial, then $\mathcal{A}$ and $S$ would both be finite, and so $\operatorname{deg}(S)=\mathbf{0} \in \operatorname{Spec}(\mathcal{A})$ in this case as well.) $\operatorname{Thus}_{\operatorname{DgSp}}(R) \subseteq \operatorname{Spec}(\mathcal{A})$, proving the theorem.

We now consider the converse of Corollary 2.2. Our two main results, Theorem 2.10 and Proposition 2.16, show that spectra of unary relations on $\mathcal{L}$ satisfy the two principal known criteria for spectra of linear orders. Theorem 2.10 is also required to complete the proof of Theorem 2.1, of course.

Definition 2.8 Let $R$ be a unary relation on $\mathcal{L}$, and $x$ a real number. We say that $R$ defines a lower cut at $x$ if there exist $a \prec b$ in $\mathcal{L}$ with $\theta(a)<x<\theta(b)$, 
such that for all $n \in(a, b)_{\mathcal{L}}, R(n)$ holds if and only if $\theta(n)<x$. Also, $R$ defines an upper cut at $x$ if $\bar{R}$ defines a lower cut at $x$.

In the case where $x$ is rational, it would be advisable to adjust this definition to allow $\theta^{-1}(x)$ to be in either $R$ or $\bar{R}$. However, we are only interested in the case of an irrational $x$.

Lemma 2.9 If $R$ is a unary relation on $\mathcal{L}$ which defines either a lower or an upper cut at an irrational number, then $D g S p_{\mathcal{L}}(R)$ is upward-closed under Turing reducibility.

Proof. Suppose $R$ defines a lower cut. Pick degrees $\mathbf{d}<_{\mathbf{T}} \mathbf{c}$ with $\mathbf{d} \in \operatorname{DgSp}_{\mathcal{L}}(\mathbf{R})$, and fix a set $C \in \mathbf{c}$. By Lemma 1.6, $(\mathcal{L}, R) \cong(\mathcal{L}, S)$ for some relation $S$ of degree d. By the isomorphism, we see that $S$ also defines a lower cut at some irrational number $x$. Pick $a, b \in \mathcal{L}$ as in Definition 2.8 for the lower cut defined by $S$. We know there exists a real $y \in(\theta(a), \theta(b))$ whose binary expansion differs from $C$ in only finitely many places, and this $y$ is irrational since $C>_{T} \emptyset$. Using a $C$-oracle (since $S \leq_{T} C$ ), we define a relation $Q$ on $\mathcal{L}$ by:

1. On $(-\infty, a]_{\mathcal{L}}$ and on $[b,+\infty)_{\mathcal{L}}, Q=S$;

2. For all $n \in(a, b)_{\mathcal{L}}$ with $\theta(n)<y, n \in Q$; and

3. For all $n \in(a, b)_{\mathcal{L}}$ with $y<\theta(n), n \notin Q$.

Then $(\mathcal{L}, S) \cong(\mathcal{L}, Q)$. Clearly $Q \leq_{T} C$, and from $Q$ we can compute the real $y$, so $C \leq_{T} Q$. Therefore $\mathbf{c} \in \operatorname{DgSp}_{\mathcal{L}}(\mathbf{R})$.

If $R$ defines an upper cut, then $\bar{R}$ defines a lower cut, so again $\operatorname{DgSp}_{\mathcal{L}}(R)=$ $\operatorname{DgSp}_{\mathcal{L}}(\bar{R})$ is upward-closed.

Theorem 2.10 For any unary relation $R$ on $\mathcal{L}$, the following are equivalent.

1. $R$ is not intrinsically computable.

2. $R$ cannot be defined by a quantifier-free formula with parameters from $\mathcal{L}$.

3. $D g S p_{\mathcal{L}}(R)$ is upward-closed under Turing reducibility.

Recall that $R$ is intrinsically computable if $\operatorname{DgSp}_{\mathcal{L}}(R)$ contains only the degree 0. More generally, for any property $\mathcal{P}$ of sets, $R$ is intrinsically $\mathcal{P}$ if $\mathcal{P}$ holds of all images of $R$ in isomorphic computable copies of $\mathcal{L}$ (see [10]). For a property $\mathcal{P}$ which is invariant under Turing equivalence, therefore, $R$ is intrinsically $\mathcal{P}$ iff $\mathcal{P}$ holds of all Turing degrees in $\operatorname{DgSp}_{\mathcal{L}}(R)$.

Proof. The implications $1 \Longrightarrow 2$ and $3 \Longrightarrow 1$ are immediate. In fact, Moses proved in [18] that $1 \Longleftrightarrow 2$. To prove that $2 \Longrightarrow 3$, fix any degrees $\mathbf{d} \leq_{\mathbf{T}} \mathbf{c}$, and suppose (using Lemma 1.6) that $S \in \mathbf{d}$ and $(\mathcal{L}, R) \cong(\mathcal{L}, S)$. Let $\mathcal{M}$ be another computable copy of $\mathcal{L}$, and fix a set $C \in \mathbf{c}$ to be our oracle. We will build a $C$-computable isomorphism $g$ from $\mathcal{L}$ onto $\mathcal{M}$, such that $g(S) \equiv_{T} C$. This will prove the upward-closure of $\operatorname{DgSp}_{\mathcal{L}}(R)$. 
In fact, it is fine for $\mathcal{M}$ to be $\mathcal{L}$ itself, but we give the two copies different names in order to distinguish them. We write $\prec_{\mathcal{L}}$ and $\prec_{\mathcal{M}}$ for the orders on the two structures. Elements of $\mathcal{L}$ will be named $a, b$, and $c$, while elements of $\mathcal{M}$ will be named $x, y$, and $z$.

The function $g$ will be extended to a larger domain $D_{s+1} \subset \mathcal{L}$ and range $W_{s+1} \subset \mathcal{M}$ at each stage $s+1$. This extension will involve two steps. During the first, we will extend $g$ to a domain $D_{s+1}^{\prime}$ and range $W_{s+1}^{\prime}$; then we extend $g$ from these to $D_{s+1}$ and $W_{s+1}$ during the second step.

Start with $g$ as the empty function, so $D_{0}=W_{0}=\emptyset$. At stage $s+1$, we first perform Step 1. Let $W_{s}=\left\{z_{1}, \ldots, z_{n}\right\}$, with each $z_{j} \prec \mathcal{M} z_{j+1}$. Set $a_{j}=g^{-1}\left(z_{j}\right)$ for each $j$. For convenience, we will think of $z_{0}$ and $a_{0}$ as being $-\infty$, i.e., to the left of all elements of $\mathcal{M}$ and $\mathcal{L}$ respectively, and $z_{n+1}$ and $a_{n+1}$ as being $+\infty$. For each $j \leq n+1$, let $x_{j}<y_{j}$ be the two least elements (under $<$ ) in the $\prec \prec_{\mathcal{M}}$-interval $\left(z_{j}, z_{j+1}\right)_{\mathcal{M}}$. Enumerate all these $x_{j}$ and $y_{j}$ (and all of $W_{s}$ ) into $W_{s+1}^{\prime}$ immediately.

Now use a $C$-oracle (which can compute $S$, since $S \leq_{T} C$ ) to search for the least pair $\langle b, c\rangle$ of elements in $\mathcal{L}-D_{s}$ satisfying:

- $b$ and $c$ lie in the same interval $\left(a_{i}, a_{i+1}\right)$, for some $i \leq n$; and

- for this $i, b \prec_{\mathcal{L}} c \Longleftrightarrow x_{i} \prec_{\mathcal{M}} y_{i}$; and

- $b \in S \Longleftrightarrow c \notin S$; and

- $b \in S \Longleftrightarrow s \in C$.

Let $c_{i}$ be this $c$, and let $b_{i}$ be the least corresponding $b$; these must exist, as we prove below. Define $g\left(b_{i}\right)=x_{i}$ and $g\left(c_{i}\right)=y_{i}$, thus enumerating $b_{i}$ and $c_{i}$ into $D_{s+1}^{\prime}$. By the conditions given, $g$ is still a partial isomorphism.

For each $j \neq i$, search for the least pair $\left\langle b_{j}, c_{j}\right\rangle$ of distinct elements in $\mathcal{L}$ satisfying:

- $b_{j}$ and $c_{j}$ both lie in the interval $\left(a_{j}, a_{j+1}\right)$; and

- $b_{j} \prec_{\mathcal{L}} c_{j} \Longleftrightarrow x_{j} \prec_{\mathcal{M}} y_{j}$; and

- $b_{j} \in S \Longleftrightarrow c_{j} \in S$.

Again, define $g\left(b_{j}\right)=x_{j}$ and $g\left(c_{j}\right)=y_{j}$, thus enumerating $b_{j}$ and $c_{j}$ into $D_{s+1}^{\prime}$. The conditions ensure that $g$ is still a partial isomorphism. $D_{s+1}^{\prime}$ now contains $D_{s}$ and all $x_{j}$ and $y_{j}$ (including $x_{i}$ and $y_{i}$ ).

To see that such elements $b_{i}$ and $c_{i}$ must exist, consider the case when $s \in C$. Then we require $b_{i} \prec c_{i}$ and $b_{i} \in S$ and $c_{i} \in \bar{S}$. If no interval $\left(a_{i}, a_{i+1}\right)_{\mathcal{L}}$ with $i \leq n$ contains such elements, then for each of these intervals there is a real number $t_{i} \in\left[\theta\left(a_{i}\right), \theta\left(a_{i+1}\right)\right]_{\mathbb{R}}$ such that for all $b \in\left(a_{i}, a_{i+1}\right)_{\mathcal{L}}$,

$$
b \in S \Longleftrightarrow \theta(b)>t_{i}
$$

If all these $t_{i}$ were rational, then $S$ (and hence $R$ ) would be definable by a quantifier-free formula using parameters $a_{1}, \ldots, a_{n}$ and $\theta^{-1}\left(t_{0}\right), \ldots, \theta^{-1}\left(t_{n+1}\right)$. 
So some $t_{i}$ must be irrational. But then $t_{i}$ must lie strictly between $a_{i}$ and $a_{i+1}$ in $\mathcal{L}$, so $S$ defines an upper cut at $t_{i}$, and by Lemma $2.9, \operatorname{DgSp}_{\mathcal{L}}(S)$ is upwardclosed and we are finished. Therefore, we may assume that some interval has no corresponding $t_{i}$ at all, and within this interval there exist elements $b_{i}$ and $c_{i}$ satisfying the given conditions. A similar analysis applies for the case where $s \notin C$. Moreover, no matter which $i \leq n$ we finally choose, for each $j \neq i$ there clearly exist $b_{j}$ and $c_{j}$ satisfying the second set of conditions, simply because $\left(a_{j}, a_{j+1}\right)_{\mathcal{L}}$ contains infinitely many elements. So we find the elements we need, which completes Step 1.

In Step 2, let $z_{1} \prec_{\mathcal{M}} \cdots \prec_{\mathcal{M}} z_{n}$ be the elements of $W_{s+1}^{\prime}$. As before, set $a_{i}=g^{-1}\left(z_{i}\right)$ for each $i \leq n$, and define $a_{0}, z_{0}, a_{n+1}$, and $z_{n+1}$ as in Step 1. For each $j \leq n$, let $x_{j}$ be the least element (under $<$ ) of the interval $\left(z_{j}, z_{j+1}\right)_{\mathcal{M}}$, and let $W_{s+1}=W_{s+1}^{\prime} \cup\left\{x_{0}, \ldots, x_{n}\right\}$. For each $j \leq n$, find the least element $b_{j}$ (under $<$ ) in $\left(a_{j}, a_{j+1}\right)_{\mathcal{L}}$, and define $g\left(b_{j}\right)=x_{j}$. Thus all these $b_{j}$ enter $D_{s+1}$. This completes Step 2, and stage $s+1$.

We now claim that the map $g$ built by this process is the isomorphism we required. First, the entire construction is $C$-computable, and $g$ is never redefined on any domain element, so $g \leq_{T} C$. Next, $g$ is total (by Step 2, which ensures that the least element not in $D_{s}$ must enter $D_{s+1}$ ) and onto (since Step 1 enumerates into $W_{s+1}$ the least element not in $W_{s}$ ). At every step we ensure that $g$ respects $\prec_{L}$ and $\prec_{\mathcal{M}}$, by choosing domain elements only from the appropriate intervals in $\mathcal{L}$. So $g$ is a $C$-computable isomorphism.

That $g(S) \leq_{T} C$ is immediate, since both $g$ and $S$ are $C$-computable. To compute from $g(S)$ whether $s \in C$, we need only consider the elements $z_{1} \prec_{\mathcal{M}}$ $\cdots \prec_{\mathcal{M}} z_{n}$ of $W_{s}$, along with $z_{0}$ and $z_{n+1}$ as above, and find the unique interval $\left(z_{i}, z_{i+1}\right)_{\mathcal{M}}$ such that of the two <-least elements $x_{i} \prec_{M} y_{i}$ of that interval, one lies in $g(S)$ and the other does not. The choice of $b_{i}$ and $c_{i}$ in the construction guarantees that

$$
s \in C \Longleftrightarrow b_{i} \in S \Longleftrightarrow x_{i} \in g(S),
$$

so our $g(S)$-oracle determines whether $s \in C$. The key to this coding is the fact that the sets $W_{s}$ (or more specifically, canonical indices for these sets, as described in [20], p. 33) are computable uniformly in $s$. The construction ensured this, by defining $W_{s+1}^{\prime}$ in Step 1 using only $W_{s}$ (i.e., without any $C$ oracle, and without knowing $D_{s}$ or $g$ ), and then by defining $W_{s+1}$ similarly from $W_{s+1}^{\prime}$ in Step 2. Thus $C \equiv_{T} g(S)$, and so c lies in $\operatorname{DgSp}_{\mathcal{L}}(S)=\operatorname{DgSp}_{\mathcal{L}}(R)$.

The proof of Theorem 2.10 is based on the fact that in $\mathcal{L}$, given a finite set of parameters (such as $W_{s}$, in Step 1), there are only finitely many 1-types over those parameters. In Step 1, for each of these types over $W_{s}$ (except those realized by the parameters themselves), we added to $W_{s+1}^{\prime}$ two new elements realizing that type. Then in Step 2, we added to $W_{s+1}$ one new element realizing each 1-type over $W_{s+1}^{\prime}$ (again excepting those types realized by the parameters themselves). By induction, therefore, each $W_{s}$ is finite, allowing this process to continue.

The same process could be used with $n$-types, for any $n \geq 1$. In Step 1 we would enumerate elements $x_{1}, \ldots x_{n}, y_{1}, \ldots, y_{n}$ into $W_{s+1}^{\prime}$ for each $n$-type over 
$W_{s}$, so that the two $n$-tuples $\vec{x}$ and $\vec{y}$ both realize that $n$-type. (We would not include $n$-types realized by $n$-tuples of parameters.) Again, given that $R$ is not definable without quantifiers, there is at least one of these types for which we can find a pre-image in $R$ for $\vec{x}$ and a preimage in $\bar{R}$ for $\vec{y}$, and define $f$ so as to code $C$ into $f(R)$. We repeat Step 2 similarly with $n$-tuples for the finitely many $n$-types over $W_{s+1}^{\prime}$. This yields the analogue of Theorem 2.10 for $n$-ary relations:

Corollary 2.11 A finitary relation $R$ on $\mathcal{L}$ is not intrinsically computable $\Longleftrightarrow$ $R$ is not definable by a quantifier-free formula with parameters $\Longleftrightarrow D g S p_{\mathcal{L}}(R)$ is upward-closed under Turing reducibility.

Corollary 2.12 Let $\mathcal{P}$ be any collection of Turing degrees which is bounded above under $\leq_{T}$. (For instance, $\mathcal{P}$ could be the $\Sigma_{1}^{0}$ degrees, or the $\Delta_{\omega}^{0}$ degrees, or the hyperarithmetical degrees.) Then every finitary relation on $\mathcal{L}$ which is intrinsically $\mathcal{P}$ must be intrinsically computable.

Corollary 2.13 For every finitary relation $R$ on $\mathcal{L}$, there exists a structure $\mathcal{S}$ such that $\operatorname{Spec}(\mathcal{S})=D g S p_{\mathcal{L}}(R)$.

Proof. If $R$ is intrinsically computable, let $\mathcal{S}$ be any trivial structure in a finite language. Otherwise, the corollary follows from Corollary 2.11 and Sublemma 2.14 below.

Sublemma 2.14 Let $R$ be any finitary relation on any computable, relatively computably categorical structure $\mathcal{A}$ (as defined on $p$. 5) in any finite language. If $D_{g S p}(R)$ is upward-closed, then there exists a structure $\mathcal{S}$ such that $\operatorname{Spec}(\mathcal{S})=$ $D g S p_{\mathcal{A}}(R)$.

Proof. Let $\mathcal{S}=(\mathcal{A}, R)$, a structure in the language of $\mathcal{A}$ with one additional predicate $R$. Clearly $\operatorname{DgSp}_{\mathcal{A}}(R) \subseteq \operatorname{Spec}(\mathcal{S})$. For the reverse inclusion, take any structure $\mathcal{M} \cong \mathcal{S}$, and let $\mathcal{M}^{\prime}$ be the reduct of $\mathcal{M}$ to the language of $\mathcal{A}$. By relative computable categoricity, there is an $\mathcal{M}^{\prime}$-computable (hence $\mathcal{M}$ computable) isomorphism $f$ taking $\mathcal{M}^{\prime}$ onto $\mathcal{A}$. Let $S=f\left(R^{\mathcal{M}}\right)$; then $S \leq_{T} \mathcal{M}$ and $\operatorname{deg}(S) \in \operatorname{DgSp}_{\mathcal{A}}(R)$, so by upward-closure $\operatorname{deg}(\mathcal{M}) \in \operatorname{DgSp}_{\mathcal{A}}(R)$ as well.

We note one more theorem about spectra of linear orders, and show that the same result holds for unary relations on $\mathcal{L}$. The theorem is from Richter in [19]:

Theorem 2.15 (Richter) If $\mathcal{A}$ is a linear order such that the degree $\mathbf{0}$ does not lie in $\operatorname{Spec}(\mathcal{A})$, then $\operatorname{Spec}(\mathcal{A})$ does not contain a least degree. Indeed $\operatorname{Spec}(\mathcal{A})$ contains a minimal pair of degrees.

This result also applies to other structures, such as trees, which satisfy a condition given by Richter on effective extendability of embeddings.

Proposition 2.16 If $R$ is a unary relation on $\mathcal{L}$ such that the degree $\mathbf{0}$ does not lie in $D g S p_{\mathcal{L}}(R)$, then $D g S p_{\mathcal{L}}(R)$ does not contain a least degree. Indeed $D g S p_{\mathcal{L}}(R)$ contains a minimal pair of degrees. 
Proof. Our proof simply adapts Richter's proof of Theorem 2.15 in [19] to spectra of relations on $\mathcal{L}$, building a unary relation $S \subset \omega$ and an isomorphism $h:(\mathcal{L}, S) \rightarrow(\mathcal{L}, R)$ such that the infimum $\operatorname{deg}(R) \wedge \operatorname{deg}(S)$ of the degrees of $R$ and $S$ under Turing reducibility is the degree $\mathbf{0}$. (That is, $R$ and $S$ form a minimal pair.) We define one-to-one functions $h_{s}$ from finite sets $B_{s}$ into $\mathcal{L}$, respecting $\prec$ and with $h_{s} \subseteq h_{s+1}$, such that $\cup_{s} B_{s}=\omega(=|\mathcal{L}|)$ and $h=\cup_{s} h_{s}$ is a bijection. We write $S_{s}=h_{s}^{-1}(R) \subseteq B_{s}$. At stage 0 , let $B_{0}$ be the empty set and $h_{0}$ the empty map.

At stage $s+1$, we attempt first to satisfy a minimal-pair requirement for $R$ and $S$. Pick $i$ and $j$ such that $s=\langle i, j\rangle$, and ask whether there exist $\sigma \in 2^{<\omega}$, $x \in \omega$, and an embedding $\alpha:\left(\operatorname{dom}(\sigma), \prec, \sigma^{-1}(1)\right) \rightarrow(|\mathcal{L}|, \prec, R)$ such that $\operatorname{lh}(\sigma)>\max B_{s}$ and $\sigma \mid B_{s}=S_{s}$ and $\Phi_{i}^{\sigma}(x) \neq \Phi_{j}^{R}(x)$. If not, we let $h_{s+1}^{\prime}=h_{s}$ and $S_{s+1}^{\prime}=S_{s}$. If so, we pick the shortest such $\sigma$ and the corresponding $\alpha$ and let $S_{s+1}^{\prime}=\sigma^{-1}(1)$ and $h_{s+1}^{\prime}=\alpha$.

Next, to make $h$ a bijection, we pick the least number $z \notin \operatorname{range}\left(h_{s+1}^{\prime}\right)$ and the least $y \notin \operatorname{dom}\left(h_{s+1}^{\prime}\right)$ such that $y$ lies in the appropriate interval under $\prec$, and let $B_{s+1}=\operatorname{dom}\left(h_{s+1}^{\prime}\right) \cup\{y\}$ and $h_{s+1}=h_{s+1}^{\prime} \cup\{\langle y, z\rangle\}$. This completes the construction.

Clearly this construction builds an automorphism $h=\cup_{s} h_{s}$ of $\mathcal{L}$. (To see that $h$ has domain $|\mathcal{L}|$, notice that at infinitely many stages we will find the $\sigma$, $x$, and $\alpha$ for which we search, and $\operatorname{dom}\left(h_{s}\right)$ will be extended to a longer initial segment of $\omega$ at each such stage.) We define $S=h^{-1}(R)=\cup_{s} S_{s}$, yielding $\operatorname{deg}(S) \in \operatorname{DgSp}_{\mathcal{L}}(R)$. To see that $\operatorname{deg}(R) \wedge \operatorname{deg}(S)=\mathbf{0}$, suppose that $g=\Phi_{i}^{S}=$ $\Phi_{j}^{R}$ is any total function. To compute $g(x)$, we consider the situation of the construction at stage $s+1$, where $s=\langle i, j\rangle$. The set $B_{s}$ and the function $h_{s}$ are finite, so we may use them and search for a string $\sigma \in 2^{<\omega}$ with $\operatorname{lh}(\sigma)>\max B_{s}$ and an embedding $\alpha:\left(\operatorname{dom}(\sigma), \prec, \sigma^{-1}(1)\right) \rightarrow(|\mathcal{L}|, \prec, R)$ such that $\sigma\left\lceil B_{s}=S_{s}\right.$ and $\Phi_{i}^{\sigma}(x) \downarrow$. Such $\sigma$ and $\alpha$ must exist, since any sufficiently long initial segment of $S$ could serve as $\sigma$, with $\alpha=h \uparrow \operatorname{dom}(\sigma)$. So eventually we find such a $\sigma$. It may not in fact be an initial segment of $S$, but we know from our construction that for every such $\sigma$ and $\alpha$ and for every $x, \Phi_{i}^{\sigma}(x)=\Phi_{j}^{R}(x)$. (If not, we would have chosen $h_{s+1}^{\prime}$ and $S_{s+1}^{\prime}$ to violate this equality.) Therefore $g(x)=\Phi_{i}^{\sigma}(x)$, and $g$ is indeed computable. Hence the least degree of $\operatorname{DgSp}_{\mathcal{L}}(R)$, if it existed, would have to be $\mathbf{0}$, but $\mathbf{0} \notin \operatorname{DgSp}_{\mathcal{L}}(\mathbf{R})$.

Corollary 2.17 If $R$ is a unary relation on $\mathcal{L}$ which is not intrinsically computable, then there is an infinite subset $\mathbf{D} \subseteq D g S p_{\mathcal{L}}(\mathbf{R})$ such that every pair of distinct degrees in $\mathbf{D}$ is a minimal pair.

Proof. If $\operatorname{DgSp}_{\mathcal{L}}(R)$ contains the degree $\mathbf{0}$, then Theorem 2.10 gives the result. Otherwise, for any finite $k$ with $(\mathcal{L}, R) \cong\left(\mathcal{L}, R_{i}\right)$ for all $i \leq k$, an easy modification of the proof of Proposition 2.16 allows us to build $S$ such that $(\mathcal{L}, S) \cong(\mathcal{L}, R)$ and $\operatorname{deg}\left(R_{1}\right) \wedge \operatorname{deg}(S)=\cdots=\operatorname{deg}\left(R_{k}\right) \wedge \operatorname{deg}(S)=\mathbf{0}$. Setting $R_{k+1}=S$, we build $\left\{\operatorname{deg}\left(R_{k}\right)\right\}_{k \in \omega}$ by induction as our $\mathbf{D}$.

Theorem 2.10 and Proposition 2.16 suggest the possibility of strengthening Corollary 2.13 by requiring $\mathcal{S}$ to be a linear order, since spectra of unary relations 
on $\mathcal{L}$ must satisfy the main theorems on spectra of linear orders. Indeed, a partial result in this direction is immediate: given an infinite relation $R$, restrict the computable order $\prec$ from $\mathcal{L}$ to $R$ to get a linear order $\mathcal{A}$. By pulling $R$ back to $\omega$ via an $R$-computable bijection, we get an $R$-computable copy of $\mathcal{A}$ with domain $\omega$. Thus $\operatorname{Spec}(\mathcal{A})$ contains some degree $\leq_{T} \operatorname{deg}(R)$, and Theorem 1.4 shows that $\operatorname{deg}(R) \in \operatorname{Spec}(\mathcal{A})$, $\operatorname{so} \operatorname{DgSp}_{\mathcal{L}}(R) \subseteq \operatorname{Spec}(\mathcal{A})$.

However, without the reverse inclusion this result is trivial (just set $\mathcal{A}=\mathcal{L}$, since $\operatorname{Spec}(\mathcal{L})$ contains every Turing degree), and the reverse inclusion need not hold for the $\mathcal{A}$ built by this process. For intrinsically computable infinite relations $R$, this is immediate; for instance, let $R$ be a closed interval of $\mathcal{L}$. To emphasize the difficulties, however, we provide a more interesting example.

Let $R$ be a relation on $\mathcal{L}$ such that $\theta(R)$ is the following union of intervals in $(\mathbb{Q},<)$ :

$$
\theta(R)=\left(-1,-\frac{1}{2}\right) \bigcup\left[\bigcup_{n \notin \emptyset^{\prime \prime \prime}}\left(n-\frac{1}{\pi}, n+\frac{1}{2}\right)\right] \bigcup\left[\bigcup_{n \in \emptyset^{\prime \prime \prime}}\left[n, n+\frac{1}{2}\right)\right] .
$$

Thus, the leftmost interval of $\theta(R)$ is $\left(-1,-\frac{1}{2}\right)$. The next is either $\left(-\frac{1}{\pi}, \frac{1}{2}\right)$ if $0 \notin \emptyset^{\prime \prime \prime}$, or $\left[0, \frac{1}{2}\right)$ if $0 \in \emptyset^{\prime \prime \prime}$. The next is either $\left(1-\frac{1}{\pi}, \frac{3}{2}\right)$ or $\left[1, \frac{3}{2}\right)$ depending on whether $1 \in \emptyset^{\prime \prime \prime}$, and so on. ( $\frac{1}{\pi}$ is used simply because we need a convenient computable irrational number less than $\frac{1}{2}$.) Now the restriction of $\prec$ to $R$ gives a copy of the countable dense linear order. This is the $\mathcal{A}$ built from $R$ by the process described above, and its spectrum contains every Turing degree. However, $\operatorname{DgSp}_{\mathcal{L}}(R)$ cannot contain $\mathbf{0}$, as seen by the following result.

Proposition 2.18 For the relation $R$ on $\mathcal{L}$ described above, $D g S p_{\mathcal{L}}(R)$ is the set of all those degrees whose jump computes $\mathbf{0}^{\prime \prime}$.

A degree $\mathbf{c}$ is high if $\mathbf{c}^{\prime}=\mathbf{0}^{\prime \prime}$. (Some definitions require $\mathbf{c} \leq_{\mathbf{T}} \mathbf{0}^{\prime}$ as well.) So $\operatorname{DgSp}_{\mathcal{L}}(R)$ might be said to contain those degrees which are high-or-above. This set is certainly upward-closed under $\leq_{T}$, but it is not known whether there exists a linear order with this spectrum. Indeed, this proposition and Corollary 2.13 constitute the first proof of the following:

Corollary 2.19 There exists a structure $\mathcal{S}$ whose spectrum contains precisely those degrees $\mathbf{c}$ with $\mathbf{c}^{\prime} \geq_{\mathbf{T}} \mathbf{0}^{\prime \prime}$.

This complements a recent result in [7], which proves the existence, for each $n \in \omega$, of a structure whose spectrum contains exactly the non-low ${ }_{n}$ degrees, i.e., those degrees $\mathbf{c}$ with $\mathbf{c}^{(\mathbf{n})}>_{\mathbf{T}} \mathbf{0}^{(\mathbf{n})}$. Moreover, we can use an arbitrary degree $\mathbf{d}$ in place of $\mathbf{0}^{\prime \prime}$ in the following proof, thereby building structures with spectrum $\left\{\mathbf{c}: \mathbf{c}^{\prime} \geq \mathbf{T} \mathbf{d}\right\}$.

Proof of Proposition 2.18. We will show that $\mathbf{c}$ is in $\operatorname{DgSp}_{\mathcal{L}}(R)$ iff $\emptyset^{\prime \prime \prime} \leq{ }_{1} \operatorname{Fin}^{S}$ for some set $S \in \mathbf{c}$. This latter condition is equivalent to $\emptyset^{\prime \prime} \leq_{T} S^{\prime}$. (See Theorem III.2.3(v) and Section IV.4 of [20] for this result. By definition Fin ${ }^{S}=$ $\left\{e: W_{e}^{S}\right.$ is finite $\}$, where $W_{e}^{S}$ is the $e$-th set c.e. in the oracle $S$ under a standard enumeration.) 
For the forward inclusion, let $(\mathcal{L}, R) \cong(\mathcal{L}, S)$, with $\prec$ being the computable order of $\mathcal{L}$ as always. (By Lemma 1.6, we need only consider $\mathcal{L}$.). We will define a 1-reduction $h$ of $\emptyset^{\prime \prime \prime}$ to $\mathrm{Fin}^{S}$.

Fix an ordering of $\omega^{<\omega}$ in order type $\omega$. A string $\sigma \in \omega^{2 n+1}$ is $\prec$-increasing if $\sigma(0) \prec \sigma(1) \prec \cdots \prec \sigma(2 n)$, and $\sigma$ is $S$-alternating if for all $i \leq 2 n$ :

$$
\sigma(i) \in S \Longleftrightarrow i \text { is even. }
$$

(In the above, "even" may not be replaced by "odd.")

The number $h(n)$ will be the index of an oracle Turing functional $\Phi$ which performs the following algorithm on input $x$ and oracle $X$. First it searches for the least $\sigma_{0} \in \omega^{2 n+1}$ (according to our ordering of $\omega^{<\omega}$ ) which is $\prec$-increasing and $X$-alternating. Then, by induction, having found $\sigma_{y}$, it searches for the least $\sigma_{y+1} \in \omega^{2 n+1}$ which is $\prec$-increasing and $X$-alternating and satisfies $\sigma_{y+1}(2 n) \prec$ $\sigma_{y}(2 n)$. If it ever finds $\sigma_{x}$ in this process, it terminates and outputs 0; otherwise it diverges. (Thus the domain of $\Phi^{X}$ will always be an initial segment of $\omega$.)

Now if $n \in \emptyset^{\prime \prime \prime}$, then the $(n+1)$-st interval of $S$ in $\mathcal{L}$ includes its left end point $l$. Hence there exist $\prec$-increasing $S$-alternating strings $\sigma \in \omega^{2 n+1}$ with $\sigma(2 n)=l$, and for some $x, \sigma_{x}$ will be the least such string. Then $\Phi_{h(n)}^{S}$ will diverge on all inputs $>x$, since no $\sigma_{x+1}$ satisfying our demands will ever be found. Hence $h(n) \in \operatorname{Fin}^{S}$.

On the other hand, if $n \notin \emptyset^{\prime \prime \prime}$, then the $(n+1)$-st interval of $S$ has no left end point. In this case, for every $\sigma_{x}$ we find, there will be another sequence $\sigma_{x+1}$ found subsequently. (Just consider $\left\langle\sigma(0), \ldots, \sigma(2 n-1), l^{\prime}\right\rangle$, for any $l^{\prime} \prec \sigma(2 n)$ in the $(n+1)$-st interval of $S$.) Hence for this $n$ we will have $h(n) \notin \operatorname{Fin}^{S}$; indeed $h(n) \in \operatorname{Tot}^{S}$. Thus $h$ is a computable 1-reduction of $\emptyset^{\prime \prime \prime}$ to $\operatorname{Fin}^{S}$.

For the reverse inclusion, let $C$ be any set with $\emptyset^{\prime \prime \prime} \leq_{1} \operatorname{Fin}^{C}$ via some function $h$. We build a unary relation $S$ on $(\mathbb{Q},<)$ with $S \equiv_{T} C$ and $\left(\mathcal{L}, \theta^{-1}(S)\right) \cong(\mathcal{L}, R)$.

The right end point of the $n$-th interval of $S$ will be $r_{n}=n-\frac{1}{2}$. The leftmost interval of $S$ is $\left(-1, r_{0}\right)$. We show here how to construct the next interval of $S$, which will have a (possibly irrational) left end point $l_{1}=\lim _{s} l_{1, s}$; all other intervals are analogous. At stage 0 we enumerate $\left[0, r_{1}\right)$ into $S$ and set $l_{1,0}=0$. At stage $s+1$, we use our $C$-oracle to check whether any elements entered $W_{h(0)}^{C}$ at stage $s+1$. If not, we do nothing. If so, let $l_{1, s+1}$ be the first rational number $l$ we find such that:

(1) $-\frac{1}{4}<l<l_{1, s}$; and

(2) $\{\theta(0), \ldots \theta(s)\} \cap\left[l, l_{e, s}\right)=\emptyset$; and

(3) The binary expansion of $l$ is the same as that of $l_{1, s}$ up through the first repetition of the repeating part of $l_{1, s}$ (where, if $l_{1, s}$ is dyadic, we use the binary expansion with infinitely many 1 's); and

(4) Let $\langle i, j\rangle=\left|W_{h(0), s}^{C}\right|$, so $\langle i, j\rangle$ is the number of times we have already moved $l_{1, s}$. Consider the $i$-th element $\sigma$ in some fixed enumeration of the nonempty strings in $2^{<\omega}$. This $\sigma$ must not be the repeating part of any binary expansion of $l$. 
(The last two items will ensure that if $W_{h(0)}^{C}$ is infinite, then $\lim _{s} l_{1, s}$ will be irrational.) Such an $l_{1, s+1}$ must exist, and we enumerate all of $\left[l_{1, s+1}, l_{1, s}\right)$ into $S$. This completes the construction.

We let $l_{n}=\inf _{s} l_{n, s}$ for each $n>0$, with $l_{0}=-1$. These are the left end points of the intervals of $S$. For each $n$, if $n \in \emptyset^{\prime \prime \prime}$, then $h(n) \in \mathrm{Fin}^{C}$, so $l_{n, s}$ is only redefined finitely many times, and the $(n+1)$-st interval of $S$ is $\left[l_{n}, r_{n}\right)$, with $l_{n}=l_{n, s}$ for some $s$.

On the other hand, if $n \notin \emptyset^{\prime \prime \prime}$, then $h(n) \notin \mathrm{Fin}^{C}$, so $l_{n, s}$ was redefined infinitely many times. In this case $l_{n}$ never entered $S$ (and indeed will be shown below to be irrational), so the $(n+1)$-st interval of $S$ is the open interval $\left(l_{n}, r_{n}\right)$. To see that in this case the real number $l_{n}$ must be irrational, suppose it were rational. Then some binary string $\sigma$ would repeat forever beyond a certain digit in a fixed binary expansion of $l_{n}$. Choose an $s$ such that $l_{n}$ agrees with $l_{n, s}$ through the first appearance of $\sigma$ in the repeating part of $l_{n}$. Then every subsequent $l_{n, t}$ also agrees with that much of $l_{n}$, by requirement (3). However, eventually we reach another stage $t^{\prime}$ at which requirement (4) ensures that $\sigma$ does not appear in the repeating part of $l_{n, t^{\prime}}$, and requirement (3) subsequently ensures that the repeating part of $l_{n, t^{\prime}}$ appears in the same place in every subsequent $l_{n, t^{\prime \prime}}$. Hence $\sigma$ cannot repeat forever in $l_{n}$, contradicting our assumption. Thus $l_{n}$ is irrational whenever $n \notin \emptyset^{\prime \prime \prime}$. This proves that $\left(\mathcal{L}, \theta^{-1}(S)\right) \cong(\mathcal{L}, R)$.

This enumeration of $S$ was $C$-computable, and no element $x$ entered $S$ at any stage after stage $\theta^{-1}(x)$, because of requirement (2). Hence $S \leq_{T} C$. But $S$ is not a finite union of intervals in $\mathcal{L}$, so by Theorem 2.10, c lies in $\operatorname{DgSp}_{\mathcal{L}}(R)$ as required.

\section{Graphs}

An (undirected) graph consists of a set of elements, called vertices or nodes, and an irreflexive symmetric binary relation (the adjacency or edge relation) on the vertices. For this class, the natural countably universal structure to use is the random graph, which by definition is the Fraïssé limit of the class of all finite graphs. (Fraïssé limits are discussed in moderate detail in the next section.) The random graph is well described in $([12], 6.4)$, and is characterized there as follows.

Theorem 3.1 ([12], p. 177) Let $\mathcal{G}$ be a countable graph. The following are equivalent.

(a) $\mathcal{G}$ is (isomorphic to) the random graph.

(b) For every pair $(X, Y)$ of disjoint finite sets of vertices of $\mathcal{G}$, there is an element $\notin X \cup Y$ which is adjacent to all vertices in $X$ but to no vertices in $Y$.

(c) For every pair $(X, Y)$ of disjoint finite sets of vertices of $\mathcal{G}$, there are infinitely many elements $\notin X \cup Y$ which are adjacent to all vertices in $X$ but to no vertices in $Y$. 
Part (c) does not appear in [12], but is clearly equivalent to part (b).

It follows from this result that every countable graph $\mathcal{B}$ embeds into the random graph, simply by mapping the vertices $b_{1}, b_{2}, b_{3}, \ldots$ of $\mathcal{B}$ one at a time to appropriate nodes of the random graph. Our specific computable copy $\mathcal{G}$ of the random graph is easily constructed. The domain of $\mathcal{G}$ is $\omega$, as usual. We fix a computable listing $\left(X_{i}, Y_{i}\right)_{i \in \omega}$ of all pairs of finite disjoint subsets $X_{i}$ and $Y_{i}$ of $\omega$, with the properties that every number in $X_{i} \cup Y_{i}$ is $<i$. The edge relation $E^{\mathcal{G}}$ simply contains all pairs $(i, x)$ and $(x, i)$ such that $x \in X_{i}$. This is computable and symmetric and clearly satisfies condition (2) of Theorem 3.1, so $\mathcal{G}$ is isomorphic to the random graph. We define

$$
Z_{i}=\left\{a \in \mathcal{G}: a \notin X_{i} \cup Y_{i} \&\left(\forall x \in X_{i}\right)(a, x) \in E \&\left(\forall y \in Y_{i}\right)(a, y) \notin E\right\} .
$$

Thus each $Z_{i}$ is the infinite computable set of nodes used to ensure that $X_{i}$ and $Y_{i}$ satisfy Theorem 3.1. Also, the Boolean combinations of sets $Z_{i}$ are precisely the subsets of $\mathcal{G}$ which are definable by quantifier-free formulas with parameters.

Theorem 3.2 The computable random graph $\mathcal{G}$ built above is spectrally universal.

Since trivial graphs have spectrum $\{\mathbf{0}\}$, we immediately get:

Corollary 3.3 Let $\mathcal{B}$ be any countable graph. Then there exists a unary relation $R$ on the random graph $\mathcal{G}$ constructed above, such that

$$
\operatorname{DgSp} p_{\mathcal{G}}(R)=\operatorname{Spec}(\mathcal{B})
$$

In concert with results by Hirschfeldt, Khoussainov, Shore, and Slinko in [11], this yields a far stronger theorem.

Theorem 3.4 (Hirschfeldt, Khoussainov, Shore, Slinko) For each nontrivial countable structure $\mathcal{S}$ (in any computable language, finite or infinite), there exists a symmetric irreflexive graph with the same spectrum as $\mathcal{S}$.

If the language is finite, this holds for trivial structures as well. Moreover, in [11] the authors prove the same result for directed graphs, partial orders, lattices, rings, integral domains of arbitrary characteristic, commutative semigroups, and two-step nilpotent groups.

Corollary 3.5 Let $\mathcal{S}$ be any nontrivial countable structure in any computable language, or any countable structure in any finite language. Then there exists a unary relation $R$ on the random graph $\mathcal{G}$ constructed above, such that

$$
D g S p_{\mathcal{G}}(R)=\operatorname{Spec}(\mathcal{S})
$$

Proof of Corollary 3.5. By Theorem 3.4, there exists a countable graph $\mathcal{B}$ such that $\operatorname{Spec}(\mathcal{B})=\operatorname{Spec}(\mathcal{S})$. Applying Corollary 3.3 gives the result. 
Proof of Theorem 3.2. Let $\mathcal{A}$ be any nontrivial countable graph, and suppose the degree $\mathbf{c}$ lies in $\operatorname{Spec}(\mathcal{A})$. Fix a set $C$ in $\mathbf{c}$ and a countable graph $\mathcal{B} \cong \mathcal{A}$ with domain $\omega$ and edge relation $F \equiv_{T} C$.

Construction. Let $\mathcal{B}_{s}$ be the substructure with domain $\{0, \ldots, s-1\} \subseteq \mathcal{B}$ under the restriction of $F$. We build a $(C$-computable) embedding $g$ of $\mathcal{B}$ into $\mathcal{G}$, starting with $R_{0}=S_{0}=\emptyset$. (The relation $R$ will be the image of $g$, and $S$ will be the complement of $R$.)

Having defined $g$ on $\mathcal{B}_{s}$, we consider the vertex $s$ of $\mathcal{B}_{s+1}$. Using a $C$-oracle, we let $g(s)$ be the least node we find in $\mathcal{G}$ which is adjacent to all nodes of $\left\{g(x): x \in \mathcal{B}_{s} \& F(x, s)\right\}$ but not adjacent to any nodes of $S_{s} \cup\{g(x): x \in$ $\left.\mathcal{B}_{s} \& \neg F(x, s)\right\}$. These two sets are finite and disjoint, so such a node $g(s)$ must exist.

Next we extend $S_{s}$ to $S_{s+1}$, a set of vertices which will not be allowed into $R$ at any future stage. For each subset $P$ of $R_{s+1} \cup S_{s}$ in turn, we choose $j$ such that $P=X_{j}$ and $\left(R_{s+1} \cup S_{s}\right)-P=Y_{j}$, and search for the least $a_{P} \in Z_{j}$. Since $\mathcal{G}$ is the random graph, we eventually find such an $a_{P}$, and (for each $P$ ) we enumerate $a_{P}$ into $S_{s+1}$.

It is clear that this process constructs an embedding $g$ of $\mathcal{B}$ into $\mathcal{G}$, with image $R=\cup_{s} R_{s}$. Moreover, $S=\cup_{s} S_{s}$ is precisely the complement of $R$, since any vertex $x$ which never enters $R$ must eventually be chosen as $a_{P}$ for some $P$, at or before the stage $s+1$ when all vertices $<x$ have entered $R_{s+1} \cup S_{s}$. We also note that $R \neq Z_{i}$ for every $i$, since once we reach a stage $s+1>i$ we have $X_{i} \cup Y_{i} \subseteq R_{s} \cup S_{s}$, so an element of $Z_{i}$ will be enumerated into $S_{s+1}$. This shows that $R$ cannot be defined in $\mathcal{G}$ by any quantifier-free formula with parameters.

Since $R$ and $S(=\bar{R})$ are both computably enumerable in $C$, we have $R \leq_{T} C$. We have not bothered to code $C$ into $R$ in this construction; one could do so, but instead we cite Proposition 3.6 and Lemma 3.8 below to see that $\mathbf{c}$ lies in $\operatorname{DgSp}_{\mathcal{G}}(R)$. As in the proof of Theorem 2.1, we note that Proposition 3.6 can be applied uniformly. That is, with a $C$-oracle, one could build $R \equiv_{T} C$ uniformly in an index $e$ such that $F=\Phi_{e}^{C}$ gives the edge relation on $\mathcal{B}$.

As always, in order to demonstrate that $\operatorname{Spec}(\mathcal{A}) \subseteq \operatorname{DgSp}_{\mathcal{G}}(R)$, we must now show that if $\mathcal{B}$ and $\tilde{\mathcal{B}}$ are isomorphic undirected graphs of degree $\mathbf{c}$ and $\tilde{\mathbf{c}}$ respectively, then the relations $R$ and $\tilde{R}$ built by this process satisfy $(\mathcal{G}, R) \cong$ $(\mathcal{G}, \tilde{R})$. Since the maps $g$ and $\tilde{g}$ embed $\mathcal{B}$ and $\tilde{\mathcal{B}}$ into $\mathcal{G}$ with images $R$ and $\tilde{R}$, we immediately have an isomorphism $\rho: R \rightarrow \tilde{R}$. We extend $\rho$ to an automorphism of $\mathcal{G}$ by a back-and-forth process, one node at a time, as follows.

Assume that $\rho$ is already defined on all of $R$ and on finitely many nodes $s_{1}, \ldots, s_{m}$ of $S=\bar{R}$. Take the least node $x \in S$ not yet in the domain of $\rho$, and choose $s$ such that $x$ entered $S$ at stage $s+1$ in the construction of $g$ above. Now, by the construction, no node adjacent to $x$ could have entered $R$ at any stage after $s+1$, so $x$ is adjacent to only finitely many vertices $r_{1}, \ldots, r_{n}$ in $R$. Choose a stage $t$ such that $\rho\left(r_{1}\right), \ldots, \rho\left(r_{n}\right) \in \tilde{R}_{t+1}$ and $\rho\left(s_{1}\right), \ldots, \rho\left(s_{m}\right) \in \tilde{S}_{t}$. Let

$$
P=\left\{\rho\left(r_{i}\right): i \leq n\right\} \cup\left\{\rho\left(s_{i}\right): i \leq m \& x \text { is adjacent to } s_{i}\right\} .
$$

The construction of $\tilde{g}$ chose a node $a_{P} \notin \tilde{S}_{t}$ at stage $t+1$ and enumerated 
$a_{P} \in \tilde{S}_{t+1}$, and we define $\rho(x)=a_{P}$. This extension of $\rho$ is still a partial automorphism of $\mathcal{G}$. (Since $\rho(x)=a_{P} \in \tilde{S}_{t+1}$, we know that no node of $\tilde{R}-\tilde{R}_{t+1}$ can be adjacent to $\rho(x)$; and for nodes in $\tilde{R}_{t+1} \cup \tilde{S}_{t}$, the choice of $P$ gives the result.) Moreover, the extension maps $x \in S$ to $\rho(x) \in \tilde{S}$, as required.

Iterating this process by the standard back-and-forth approach yields an automorphism of $\mathcal{G}$ mapping $R$ onto $\tilde{R}$, so indeed $\operatorname{deg}(\tilde{R}) \in \operatorname{DgSp}_{\mathcal{G}}(R)$, and $\operatorname{Spec}(\mathcal{A}) \subseteq \operatorname{DgSp}_{\mathcal{G}}(R)$. Conversely, let $(\tilde{\mathcal{G}}, \tilde{R}) \cong(\mathcal{G}, R)$ with $\tilde{\mathcal{G}}$ computable. Then the edge relation on $\tilde{\mathcal{G}}$ is computable, and its restriction to $\tilde{R}$ yields a copy of $\mathcal{A}$ with domain $\tilde{R}$. To get an $\tilde{R}$-computable copy of $\mathcal{A}$ with domain $\omega$, we simply pull this copy back to $\omega$ via an $\tilde{R}$-computable bijection. Thus $\operatorname{Spec}(\mathcal{A})$ contains a degree $\leq_{T} \tilde{R}$, and hence must contain $\operatorname{deg}(\tilde{R})$ itself, by Theorem 1.4, since $\mathcal{A}$ is nontrivial. So $\operatorname{DgSp}_{\mathcal{G}}(R) \subseteq \operatorname{Spec}(\mathcal{A})$, and the two spectra are equal.

In the case of graphs, we can prove the converse of Corollary 3.3 as well. Again, Theorem 3.4 is of use; we simply pass to the language of graphs with an additional unary relation. Given a unary relation $R$ on $\mathcal{G}$, we immediately have a structure $\mathcal{H}=(\mathcal{G}, R)$ in this language, whose spectrum will be precisely $\operatorname{DgSp}_{\mathcal{G}}(R)$, by Propositions 3.6 and 3.9 below. We then appeal to Theorem 3.4 to show that there is a graph with the same spectrum. The actual converse is Proposition 3.9; everything before that leads up to its proof. We note that Proposition 3.9 becomes an open question when stated for linear orders instead of graphs.

Also, unlike for linear orders, the restriction to nontrivial structures in Definition 1.1 is necessary for graphs. There do exist infinite trivial graphs, such as the complete graph on countably many vertices, and using Lemma 3.8, one sees that it is impossible for any image of such a graph to have degree spectrum $\{\mathbf{0}\}$ as a relation on $\mathcal{G}$.

Proposition 3.6 Let $R$ be a relation on our computable copy $\mathcal{G}$ of the random graph. If $R$ is not intrinsically computable, then $D g S p_{\mathcal{G}}(R)$ is upward-closed under Turing reducibility.

Proof. We give the full proof for a unary relation $R$. The proof is substantially analogous to that of Theorem 2.10. Fix any degrees $\mathbf{d} \leq_{\mathbf{T}} \mathbf{c}$, and suppose (using Lemma 1.6) that $S \in \mathbf{d}$ and $(\mathcal{G}, R) \cong(\mathcal{G}, S)$. Also, fix a set $C \in \mathbf{c}$ to be our oracle.

Let $\mathcal{H}$ be another computable copy of the random graph. We will build an isomorphism $g$ from $\mathcal{G}$ to $\mathcal{H}$ such that $C \leq_{T} g(S)$. Moreover, $g$ will be $C$-computable, forcing $g(S) \leq_{T} C$. This will prove the proposition. (One can just take $\mathcal{H}=\mathcal{G}$, but we will give the two copies different names, to distinguish the domain of $g$ from the range. For clarity, $a, b$, and $c$ will represent nodes in $\mathcal{G}$, while $x, y$, and $z$ represent nodes in $\mathcal{H} . E^{\mathcal{G}}$ and $E^{\mathcal{H}}$ will denote the edge relations on the two graphs.)

For any finite partial binary function $\sigma: \mathcal{H} \rightarrow 2$, we define the computable set $Z_{\sigma} \subset \mathcal{H}$ (by analogy to $Z_{i}$, from p. 19):

$$
Z_{\sigma}=\left\{x \in \mathcal{H}:(\forall y \in \operatorname{dom}(\sigma))\left[\sigma(y)=1 \Longleftrightarrow(x, y) \in E^{\mathcal{H}}\right]\right\} .
$$


Also, we define the computable subset $B_{\sigma}$ of $\mathcal{G}$ :

$$
B_{\sigma}=\left\{a \in \mathcal{G}:(\forall y \in \operatorname{dom}(\sigma))\left[\sigma(y)=1 \Longleftrightarrow\left(a, g^{-1}(y)\right) \in E^{\mathcal{G}}\right]\right\} .
$$

So, if $x \in Z_{\sigma}$ and we are searching for an element to be its preimage $g^{-1}(x)$, we need to choose that element from $B_{\sigma}$ (assuming that $\operatorname{dom}(\sigma)$ is already contained in the range of $g$ ).

The strategy is as follows. At the start of each stage, $g$ is a partial isomorphism from $\mathcal{G}$ into $\mathcal{H}$, with some finite domain $D_{s}$ and range $W_{s}$. We partition $\mathcal{H}$ into computable subsets $Z_{\sigma}$, with $\sigma$ ranging over all binary functions with domain $W_{s}$, and build $W_{s+1} \supset W_{s}$ containing exactly two new elements from each $Z_{\sigma}$. For all but one such $\sigma$, those two elements will lie either both in $S$, or both in $\bar{S}$. The two elements for the unique remaining $\sigma$ will be used for our coding of $C$ into $g(S)$, as we extend $g$ to a partial isomorphism with range $W_{s+1}$.

We start with $g$ as the empty function, with domain $D_{0}$ and range $W_{0}$ both empty. For every $s$, stage $s+1$ consists of two steps. In Step 1, we will extend $g$ to a partial isomorphism with domain $D_{s+1}^{\prime}$ and range $W_{s+1}^{\prime}$, and then in Step 2 we extend $g$ further, to domain $D_{s+1}$, and range $W_{s+1}$.

To begin Step 1 , let $\sigma_{1}, \ldots, \sigma_{k}$ be all binary functions (in lexicographic order) whose domain is precisely the range $W_{s}$ of $g$. For every $j \leq k$, starting with $j=1$, choose $x_{j}<y_{j}$ to be the two least elements of $Z_{\sigma_{j}}-W_{s}$ such that neither $x_{j}$ nor $y_{j}$ is adjacent to any $x_{m}$ or $y_{m}$ with $1 \leq m<j$. All of these elements $x_{j}$ and $y_{j}$ are immediately enumerated into $W_{s+1}^{\prime}$, since they will enter the range of $g$. We also enumerate all of $W_{s}$ into $W_{s+1}^{\prime}$ and all of $D_{s}$ into $D_{s+1}^{\prime}$.

We show below that there exists a pair $\langle b, c\rangle$ of elements of $\mathcal{G}$ and an $i \leq k$ such that:

- $b$ and $c$ both lie in $B_{\sigma_{i}}$; and

- neither $b$ nor $c$ lies in $D_{s}$; and

- $E^{\mathcal{G}}(b, c) \Longleftrightarrow E^{\mathcal{H}}\left(x_{i}, y_{i}\right)$; and

- $b \in S \Longleftrightarrow c \notin S$. (Here we use our $C$-oracle, since $S \leq_{T} C$.)

We find the least such pair $\langle b, c\rangle$, fix the corresponding $i$, and denote $c$ as $c_{i}$ and $b$ as $b_{i}$. Both of these now enter $D_{s+1}^{\prime}$, and we use $b_{i}$ and $c_{i}$ to help code $C$ into $g(S)$. If $s \in C$ and $b_{i} \in S$, or if $s \notin C$ and $b_{i} \notin S$, then define $g\left(b_{i}\right)=x_{i}$ and $g\left(c_{i}\right)=y_{i}$. Otherwise, define $g\left(b_{i}\right)=y_{i}$ and $g\left(c_{i}\right)=x_{i}$. Thus we have

$$
s \in C \Longleftrightarrow x_{i} \in g(S),
$$

which will be the coding of $C(s)$ into $g(S)$. The conditions on $b_{i}$ and $c_{i}$ ensure that this extension of $g$ is still a partial isomorphism.

We then act for each $j \leq k$ with $j \neq i$, starting with $j=1$. We find the least pair of elements $b_{j}$ and $c_{j}$ in $\mathcal{G}$ such that

- neither $b_{j}$ nor $c_{j}$ lies in $D_{s}$; and 
- all $a$ already enumerated into $D_{s+1}^{\prime}$ satisfy $E\left(a, b_{j}\right) \Longleftrightarrow E\left(g(a), x_{j}\right)$ and also $E\left(a, c_{j}\right) \Longleftrightarrow E\left(g(a), y_{j}\right)$; and

- $E\left(b_{j}, c_{j}\right) \Longleftrightarrow E\left(x_{j}, y_{j}\right)$; and

- $b_{j} \in S \Longleftrightarrow c_{j} \in S$.

Define $g\left(b_{j}\right)=x_{j}$ and $g\left(c_{j}\right)=y_{j}$, thus enumerating $b_{j}$ and $c_{j}$ into $D_{s+1}^{\prime}$. The conditions ensure that $b_{j}, c_{j} \in B_{\sigma_{j}}$ and that the new $g$ is still a partial isomorphism.

To see that the desired elements all exist, note first that at least one $B_{\sigma_{i}}$ must have infinite intersection with both $S$ and $\bar{S}$. If there were no such $i$, then $S$ (and hence $R$ ) would be intrinsically computable, contrary to hypothesis. (In particular, aside from finitely many elements, $S$ would be definable by a quantifier-free formula using parameters from $D_{s}$.)

Fix such an $i$. It remains to show that there exist $b_{i}$ and $c_{i}$ in $B_{\sigma_{i}}-D_{s}$, one in $S$ and the other not, such that

$$
E^{\mathcal{G}}\left(b_{i}, c_{i}\right) \Longleftrightarrow E^{\mathcal{H}}\left(x_{i}, y_{i}\right) .
$$

Suppose $x_{i}$ and $y_{i}$ are adjacent in $\mathcal{H}$. Let $a$ and $\bar{a}$ be the least elements of $S \cap\left(B_{\sigma_{i}}-D_{s}\right)$ and $\bar{S} \cap\left(B_{\sigma_{i}}-D_{s}\right)$ respectively. If $a$ and $\bar{a}$ are not adjacent in $\mathcal{G}$, then let $a^{\prime}$ be an element of $B_{\sigma_{i}}-D_{s}$ adjacent to both $a$ and $\bar{a}$. (By Theorem 3.1, such an $a^{\prime}$ must exist.) If $a^{\prime} \in S$, then $a^{\prime}$ and $\bar{a}$ are the desired elements; if $a^{\prime} \in \bar{S}$, take $a^{\prime}$ and $a$ instead. The proof when $x_{i}$ and $y_{i}$ are not adjacent is completely analogous.

The existence of $b_{j}$ and $c_{j}$ for each $j \neq i$ is a similar use of Theorem 3.1, noting that every $B_{\sigma}$, being infinite, must have infinite intersection with either $S$ or $\bar{S}$. (We also use here the fact that no $x_{j}$ or $y_{j}$ is adjacent to any $x_{m}$ or $y_{m}$ with $m \neq j$, by the choice of $x_{j}$ and $y_{j}$ above.) This completes Step 1.

In Step 2, let $\rho_{1}, \ldots, \rho_{n}$ be all binary functions whose domain is precisely $W_{s+1}^{\prime}$. For every $j \leq n$, choose the least element $z_{j}$ of $Z_{\rho_{j}}-W_{s+1}^{\prime}$ and enumerate $z_{j}$ into $W_{s+1}$. Now find the least element $a$ of $\mathcal{G}-D_{s+1}^{\prime}$, and fix the $i \leq k$ such that $a \in B_{\rho_{i}}$. Set $a_{i}=a$ and define $g\left(a_{i}\right)=z_{i}$, thus putting $a_{i}$ into $D_{s+1}$. (This will ensure that $g$ has domain $\omega$.)

Then, for every $j \neq i$ in order, starting with $j=1$, find the least $a_{j} \in$ $\mathcal{G}-D_{s+1}^{\prime}$ which lies in $B_{\rho_{j}}$ and satisfies

$$
E^{\mathcal{G}}\left(a_{j}, a_{m}\right) \Longleftrightarrow E^{\mathcal{H}}\left(z_{j}, z_{m}\right)
$$

for every $m<j$ and for $m=i$. (By Theorem 3.1, $\mathcal{G}$ contains such an $a_{j}$.) Add $a_{j}$ to $D_{s+1}$ and set $g\left(a_{j}\right)=z_{j}$. The condition above guarantees that this $g$ is still a partial isomorphism. Continue until $j=n$. This completes Step 2 and stage $s+1$.

Sublemma 3.7 The finite sets $W_{s}$ are computable uniformly in s. (Specifically, canonical indices for these sets are computable uniformly in s.) 
Proof. We use induction on $s$, starting with the empty set $W_{0}$. Let $W_{s}=$ $\left\{y_{1}, \ldots, y_{n}\right\}$, from which we can compute the list $\sigma_{1}, \ldots, \sigma_{k}$ of all binary functions with domain $W_{s}$. (So $k=2^{n}$.) During Step 1 of stage $s+1$, we define $W_{s+1}^{\prime}$ to contain $W_{s}$ and the two smallest elements of each $Z_{\sigma_{j}}-W_{s}$ satisfying computable conditions. Similarly, during Step 2, we define $W_{s+1}$ to contain all of $W_{s+1}^{\prime}$ and the least element of each $Z_{\rho_{j}}-W_{s+1}^{\prime}$. Thus the choice of each element added to $W_{s+1}$ is entirely computable, with no use of $g$ or any $S$ - or $C$-oracle, so we have an algorithm, uniform in $s$, for computing the canonical index for $W_{s+1}$.

The entire construction is $C$-computable, and for all $a \in \mathcal{G}$, once $a$ appears in $D_{s}, g(a)$ is never redefined. Thus $g$ is $C$-computable. The construction makes clear that $g$ respects the edge relation on the graphs $\mathcal{G}$ and $\mathcal{H}$. To see that $\operatorname{dom}(g)=\omega$, suppose that $a$ is the least element of $\mathcal{G}-\operatorname{dom}(g)$. Then at some stage $s$, all smaller elements will lie in $D_{s}$, and at stage $s+1$, $a$ will be chosen as $a_{i}$ in Step 2 and will enter $D_{s+1}$. Also, every $x \in \mathcal{H}$ will eventually be chosen as either $a_{j}$ or $b_{j}$ or $c_{j}$ for some $j$, since in Step 2 we always choose the least available element. Thus $g$ is bijective, hence an isomorphism from $\mathcal{G}$ to $\mathcal{H}$. Moreover, since $g$ and $S$ are both $C$-computable, the bijectivity makes $g(S) \leq_{T} C$.

To see that $C \leq_{T} g(S)$, fix any $s$. By Sublemma 3.7, we can compute $W_{s}$ uniformly in $s$, so we can compute the list $\sigma_{1}, \ldots, \sigma_{k}$ of all binary functions whose domain is $W_{s}$. Also, $Z_{\sigma_{j}}$ is computable uniformly in $j$. For each $j \leq k$, find the two least elements $x_{j}<y_{j}$ of $Z_{\sigma_{j}}-W_{s}$ satisfying the (computable) conditions for inclusion in $W_{s+1}$. By our construction, there will be exactly one $i \leq k$ such that $\left\{x_{i}, y_{i}\right\}$ intersects both $g(S)$ and $g(\bar{S})$, and our coding at the end of Step 1 ensures that

$$
s \in C \Longleftrightarrow x_{i} \in g(S) .
$$

Thus $C \leq_{T} g(S)$, proving Proposition 3.6 for unary relations $R$.

For relations of arbitrary (finite) arity, the proof is essentially the same. At each stage, we consider $n$-types instead of 1-types, with the finite set $W_{s}$ as parameters. Once again, there are only finitely many such types. We ignore those types realized by an $n$-tuple of parameters; all others are realized by infinitely many $n$-tuples from $\mathcal{H}$. Since $R$ is not intrinsically computable, we can find one of these types which is realized both by an element of $R$ and by an element of $\bar{R}$. This allows us to do the coding, and the rest of the proof proceeds exactly as for unary relations.

For the record, we add the following lemma.

Lemma 3.8 Let $R$ be a relation on the random graph $\mathcal{G}$. The following are equivalent:

(1) $R$ is intrinsically computable.

(2) There are only finitely many strings $\sigma \in 2^{<\omega}$ such that both $Z_{\sigma} \cap R$ and $Z_{\sigma} \cap \bar{R}$ are infinite. 
(3) $R$ is definable with parameters in $\mathcal{G}$.

(4) $R$ is definable by a quantifier-free formula with parameters in $\mathcal{G}$.

The equivalence of (1) and (4) for Boolean algebras was proven by Downey, Goncharov, and Hirschfeldt in [4], and the same result for linear orders was proven by Moses in [18].

Proof. (1) implies (2) because, if there were infinitely many such strings, then the proof of Proposition 3.6 would go through, precluding intrinsic computability. Next, assume (2), and pick an $n$ such that no $\sigma$ of length $\geq n$ has infinite intersection with both $R$ and $\bar{R}$. Then, except for finitely many nodes in $\mathcal{G}$, membership of $x$ in $R$ is determined by which set $Z_{\sigma}$ (with $\sigma \in 2^{n}$ ) contains $x$. Thus $R$ is definable (by a quantifier-free formula) with parameters for the nodes $0, \ldots, n-1$ and the finitely many exceptions, so (2) implies (4). (3) is equivalent to (4) because $\mathcal{G}$ has quantifier elimination, and (4) immediately implies (1).

Proposition 3.9 Let $R$ be any finitary relation on any computable copy $\tilde{\mathcal{G}}$ of the random graph. Then there is a structure (and indeed a graph) whose spectrum is precisely $D g S p_{\tilde{\mathcal{G}}}(R)$.

Proof. If $R$ is intrinsically computable, then any finite graph can serve as the required structure. Otherwise $\operatorname{DgSp}_{\tilde{\mathcal{G}}}(R)$ is upward-closed, by Proposition 3.6, and Sublemma 2.14 yields the desired structure $\mathcal{S}$. This $\mathcal{S}$ is a structure in the language of graphs with an additional relation symbol, but by Theorem 3.4, there exists a graph with the same spectrum as $\mathcal{S}$. This proves the proposition.

Theorem 3.10 Let $\mathcal{D}$ be any collection of Turing degrees. The following are equivalent:

(1) $\mathcal{D}$ is the spectrum of some countable structure in some finite language.

(2) $\mathcal{D}$ is the spectrum of some countable graph.

(3) $\mathcal{D}$ is the degree spectrum of some unary relation $R$ on the random graph $\mathcal{G}$.

(4) For every $n \geq 1, \mathcal{D}$ is the degree spectrum of some $n$-ary relation on the random graph $\mathcal{G}$.

(5) For some $n \geq 1, \mathcal{D}$ is the degree spectrum of some $n$-ary relation on the random graph $\mathcal{G}$.

Proof. (1) implies (2) by Theorem 3.4, (2) implies (3) by Corollary 3.3, (3) implies (4) by taking the $n$-ary relation to be $R \times \omega^{n-1},(4)$ implies (5) trivially, and (5) implies (1) by Proposition 3.9.

For infinite languages, almost the same statement is possible; we need only worry about singleton spectra, which correspond to trivial structures, by Theorem 1.4. Apart from these, the proof is identical to that of Theorem 3.10. 
Theorem 3.11 Let $\mathcal{D}$ be any collection of Turing degrees. The following are equivalent:

(1) $\mathcal{D}$ is the spectrum of some nontrivial countable structure in some computable language.

(2) $\mathcal{D}$ is the spectrum of some nontrivial countable graph.

(3) $\mathcal{D}$ is the degree spectrum of some unary relation $R$ on the random graph $\mathcal{G}$, such that $R$ is not intrinsically computable. (Recall that Lemma 3.8 characterizes intrinsic computability for such $R$.)

(4) For every $n \geq 1, \mathcal{D}$ is the degree spectrum of some $n$-ary relation on the random graph $\mathcal{G}$ which is not intrinsically computable.

(5) For some $n \geq 1, \mathcal{D}$ is the degree spectrum of some $n$-ary relation on the random graph $\mathcal{G}$ which is not intrinsically computable.

\section{Further Questions}

It is natural to ask whether there are spectrally universal structures for theories besides the theory of linear orders and the theory of graphs. The most obvious candidate is the theory of Boolean algebras, for which the computable atomless Boolean algebra $\mathcal{B}$ would presumably be the spectrally universal structure, if one exists. This structure is the subject of current work by Csima, Harizanov, Miller, and Montalbán in [3], who conjecture that it is indeed spectrally universal.

The random graph and the countable dense linear order are both Fraïssé limits for the theories in question. Both of these theories are $\forall$-axiomatizable, and both exist in relational languages. By definition, the Fraissé limit of the class $\mathcal{C}$ of all finite models of such a theory $T$ is a countable or finite structure $\mathcal{S}$ such that every model in $\mathcal{C}$ is isomorphic to a finite substructure of $\mathcal{S}$, and such that every isomorphism between finite substructures of $\mathcal{S}$ extends to an automorphism of $\mathcal{S}$. ( $\mathcal{S}$ is sometimes also called the universal homogeneous structure for $\mathcal{C}$.) $\mathcal{S}$ is unique up to isomorphism; its existence requires that the class $\mathcal{C}$ satisfy certain properties. The definition can be extended to nonrelational languages as well; see [12] for details.

Building on Sections 2 and 3, we ask whether in other theories $T$, the Fraïssé limit of the class of finite models of $T$ might also be spectrally universal (with the possibly-necessary additional assumption that the Fraïssé limit be computably presentable). Such Fraïssé limits seem like a natural topic for computable model theory in and of themselves, since they lend themselves to finite approximation very easily, yet we are not aware of consideration of computability and Fraïssé limits anywhere in the literature up until the current work [3].

Boolean algebras differ from graphs and linear orders in that the latter two exist in purely relational languages and are $\forall$-axiomatizable. Hence any subset of a graph is itself a graph, under the restriction of the edge relation, and similarly for linear orders. For Boolean algebras this is false, and making the language 
relational would require the use of $\forall \exists$-axioms. However, Boolean algebras are uniformly locally finite, meaning that every subset of size $n$ generates a Boolean subalgebra of size computably bounded in $n$, specifically of size $\leq 2^{2^{n}}$. This means that $\mathcal{B}$ really is the Fraïsse limit of the class of finite Boolean algebras, and makes $\mathcal{B}$ the natural next subject in the study of spectral universality.

Lemma 1.2 states an obvious corollary of spectral universality of a structure $\mathcal{S}$ for a theory $T$ : that all spectra of models of $T$ can be realized as spectra of unary relations on $\mathcal{S}$. We remind the reader that for distinct theories $T$, the set of spectra of models of $T$ may well be different. For example, the upper cone above any noncomputable degree is the spectrum of some graph, but not of any linear order, nor of any Boolean algebra, by results of Richter in [19]. Moreover, Downey and Jockusch showed in [5] that every low Boolean algebra is isomorphic to a computable one, whereas a low linear order need not have a computable copy, by results of Jockusch and Soare ([13]) and Miller ([17]). Thus the sets of possible spectra are distinct for these two theories as well. Other more trivial differences for distinct theories are easily found: for instance, the theory of infinite linear orders excludes the spectrum $\{\mathbf{0}\}$, by Theorem 1.4.

For the random graph, Section 3 answers the converse as well: all unary relations on the random graph have spectra which are realized as the spectrum of some countable graph. For the computable dense linear order $\mathcal{L}$, however, this question remains open: must the degree spectrum of a unary relation on $\mathcal{L}$ be realizable as the spectrum of a linear order? Corollary 2.13 shows that some countable structure $\mathcal{S}$ (indeed in a finite language) realizes the spectrum of any given unary relation on $\mathcal{L}$, and Theorem 2.10 and Proposition 2.16 rule out the obvious approaches for trying to build a relation whose degree spectrum is not the spectrum of any linear order.

We do note, using our results from Section 2, that the converse of Lemma 1.2 fails for $\mathcal{B}$. The proof uses the following lemma.

Lemma 4.1 For every countable linear order $\mathcal{A}$, there exists a unary relation $R$ on the Boolean algebra $\mathcal{B}$ such that $\operatorname{DgSp_{\mathcal {B}}}(R)=\operatorname{Spec}(\mathcal{A})$.

Proof. Theorem 2.1 yields a subset $M \subset \mathbb{Q}$ whose spectrum (as a relation on $(\mathbb{Q},<))$ is precisely $\operatorname{Spec}(\mathcal{A})$. We view $\mathcal{B}$ as the Boolean algebra of finite unions of left-closed, right-open intervals of rational numbers. Define

$$
R=\{x \in \mathcal{B}: x \text { is of the form }(-\infty, a) \text { with } a \in M\} .
$$

Clearly $R \equiv_{T} M$. Moreover, if $(\mathbb{Q},<, N) \cong(\mathbb{Q},<, M)$, then the analogous set $S$ will satisfy $(\mathcal{B}, S) \cong(\mathcal{B}, R)$ and $S \equiv_{T} N$. Thus $\operatorname{Spec}(\mathcal{A}) \subseteq \operatorname{DgSp}_{\mathcal{B}}(R)$.

On the other hand, for any set $S$ with $(\mathcal{B}, S) \cong(\mathcal{B}, R)$, we have $(S, \subset) \cong$ $(R, \subset) \cong \mathcal{A}$, where $\subset$ is the standard proper containment relation on $\mathcal{B}$, hence computable:

$$
x \subset y \Longleftrightarrow x \neq(x \vee y)=y .
$$

Then $\operatorname{Spec}(\mathcal{A})$ must contain $\operatorname{deg}(S)$, exactly as argued in the proof of Theorem 2.1, so $\operatorname{DgSp}_{\mathcal{B}}(R) \subseteq \operatorname{Spec}(\mathcal{A})$. (Here we have again used Lemma 1.6.) 
Corollary 4.2 There exists a unary relation $R$ on the Boolean algebra $\mathcal{B}$ such that $D g S p_{\mathcal{B}}(R)$ is not the spectrum of any Boolean algebra.

Proof. Pick a linear order $\mathcal{A}$ whose spectrum contains a low degree but does not contain 0. (By a result of Jockusch and Soare in [13], such orderings exist.) Apply Lemma 4.1 to $\mathcal{A}$ to yield a relation $R$ on $\mathcal{B}$. Then $\operatorname{DgSp}_{\mathcal{B}}(R)$ contains a low degree but not $\mathbf{0}$. However, in [5] Downey and Jockusch showed that no Boolean algebra can have such a spectrum. This proves the corollary.

The relation built in Corollary 4.2 is not a Boolean subalgebra of $\mathcal{B}$, and Antonio Montalbán has asked whether there exists a Boolean subalgebra of $\mathcal{B}$ whose spectrum (as a relation on $\mathcal{B}$ ) is not that of any Boolean algebra.

\section{References}

[1] C.J. Ash \& J.F. Knight; Computable Structures and the Hyperarithmetical Hierarchy (Amsterdam: Elsevier, 2000).

[2] J. Chisholm; The complexity of intrinsically r.e. subsets of existentially decidable models, Journal of Symbolic Logic 55 (1990), 1213-1232.

[3] B.F. Csima, V.S. Harizanov, R.G. Miller, \& A. Montalbán; Computability of Fraïssé limits, to appear.

[4] R.G. Downey, S.S. Goncharov, \& D.R. Hirschfeldt; Degree spectra of relations on Boolean algebras, Algebra and Logic 42 (2003), 105-111.

[5] R.G. Downey \& C.G. Jockusch, Jr.; Every low Boolean algebra is isomorphic to a recursive one, Proceedings of the American Mathematical Society 122 (1994), 871-880.

[6] R.G. Downey \& J.F. Knight; Orderings with $\alpha$-th jump degree $\mathbf{0}^{\alpha}$, Proceedings of the American Mathematical Society 114 (1992), 545-552.

[7] S.S. Goncharov, V.S. Harizanov, J.F. Knight, C. McCoy, R.G. Miller, \& R. Solomon; Enumerations in computable structure theory, Annals of Pure and Applied Logic, 136 (2005) 3, 219-246.

[8] V.S. Harizanov; Uncountable degree spectra, Annals of Pure and Applied Logic 54 (1991), 255-263.

[9] V.S. Harizanov; Pure computable model theory, Handbook of Recursive Mathematics, vol. 1 (Amsterdam: Elsevier, 1998), 3-114.

[10] V.S. Harizanov; Relations on computable structures, Contemporary Mathematics, (University of Belgrade, 2000), 65-81.

[11] D.R. Hirschfeldt, B. Khoussainov, R.A. Shore, \& A.M. Slinko; Degree spectra and computable dimensions in algebraic structures, Annals of Pure and Applied Logic 115 (2002), 71-113. 
[12] W. Hodges; A Shorter Model Theory (Cambridge: Cambridge University Press, 1997).

[13] C.G. Jockusch, Jr. \& R.I. Soare; Degrees of orderings not isomorphic to recursive linear orderings, Annals of Pure and Applied Logic 52 (1991), 39-64.

[14] B. Khoussainov \& R.A. Shore; Computable isomorphisms, degree spectra of relations, and Scott families, Annals of Pure and Applied Logic 93 (1998), 153-193.

[15] B. Khoussainov \& R.A. Shore; Effective Model Theory: The number of models and their complexity, Models and Computability: Invited Papers from Logic Colloquium '97, ed. S.B. Cooper \& J.K. Truss, LMSLNS 259 (Cambridge: Cambridge University Press, 1999), 193-240.

[16] J.F. Knight; Degrees coded in jumps of orderings, Journal of Symbolic Logic 51 (1986), 1034-1042.

[17] R.G. Miller; The $\Delta_{2}^{0}$-spectrum of a linear order, Journal of Symbolic Logic 66 (2001), 470-486.

[18] M.F. Moses; Relations intrinsically recursive in linear orders, Zeitschrift für Mathematische Logik und Grundlagen der Mathematik 32 (1986), 467-472.

[19] L.J. Richter; Degrees of structures, Journal of Symbolic Logic 46 (1981), 723-731.

[20] R.I. Soare; Recursively Enumerable Sets and Degrees (New York: SpringerVerlag, 1987).

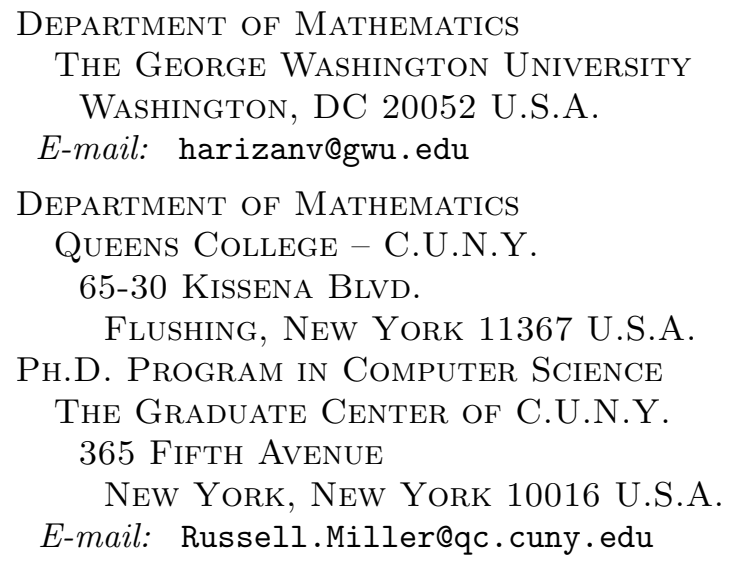

